\title{
COMPUTING THE MOMENT POLYNOMIALS OF THE ZETA FUNCTION
}

\author{
MICHAEL O. RUBINSTEIN AND SHUNTARO YAMAGISHI
}

\begin{abstract}
We describe a method to accelerate the numerical computation of the coefficients of the polynomials $P_{k}(x)$ that appear in the conjectured asymptotics of the $2 k$-th moment of the Riemann zeta function. We carried out our method to compute the moment polynomials for $k \leq 13$, and used these to experimentally test conjectures for the moments up to height $10^{8}$.
\end{abstract}

\section{INTRODUCTION}

For positive integer $k$, and any $\varepsilon>0$, Conrey, Farmer, Keating, Rubinstein, and Snaith conjectured [CFKRS] that

$$
\int_{0}^{T}|\zeta(1 / 2+i t)|^{2 k} d t=\int_{0}^{T} P_{k}(\log (t /(2 \pi))) d t+O_{k, \varepsilon}\left(T^{1 / 2+\varepsilon}\right)
$$

with the constant in the $O$ term depending on $k$ and $\varepsilon$.

In the above equation $P_{k}$ is the polynomial of degree $k^{2}$ given implicitly by the $2 k$-fold residue

$$
\begin{gathered}
P_{k}(x)=\frac{(-1)^{k}}{k !^{2}} \frac{1}{(2 \pi i)^{2 k}} \oint \cdots \oint \frac{G\left(z_{1}, \ldots, z_{2 k}\right) \Delta^{2}\left(z_{1}, \ldots, z_{2 k}\right)}{\prod_{l=1}^{2 k} z_{l}^{2 k}} \\
\quad \times e^{\frac{x}{2 \sum_{l=1}^{k} z_{l}-z_{l+k}} d z_{1} \ldots d z_{2 k},}
\end{gathered}
$$

with the path of integration over small circles about $z_{l}=0$, where

$$
\Delta\left(z_{1}, \ldots, z_{m}\right)=\prod_{1 \leq i<j \leq m}\left(z_{j}-z_{i}\right)=\left|z_{i}^{j-1}\right|_{m \times m}
$$

denotes the Vandermonde determinant,

$$
G\left(z_{1}, \ldots, z_{2 k}\right)=A_{k}\left(z_{1}, \ldots, z_{2 k}\right) \prod_{i=1}^{k} \prod_{j=1}^{k} \zeta\left(1+z_{i}-z_{j+k}\right),
$$

Received by the editor March 1, 2012 and, in revised form, May 14, 2013.

2010 Mathematics Subject Classification. Primary 11M06, 11M50; Secondary 15B52.

This work was supported by the National Science Foundation under awards DMS-0757627 (FRG grant), and an NSERC Discovery Grant. 
and $A_{k}$ is the Euler product

$$
\begin{aligned}
& A_{k}\left(z_{1}, \ldots, z_{2 k}\right) \\
& =\prod_{p} \prod_{l, j=1}^{k}\left(1-p^{-1-z_{l}+z_{k+j}}\right) \int_{0}^{1} \prod_{j=1}^{k}\left(1-\frac{e(\theta)}{p^{\frac{1}{2}+z_{j}}}\right)^{-1}\left(1-\frac{e(-\theta)}{p^{\frac{1}{2}-z_{k+j}}}\right)^{-1} d \theta .
\end{aligned}
$$

Here $e(\theta)=\exp (2 \pi i \theta)$.

We denote the coefficients of $P_{k}(x)$ by $c_{r}(k)$ :

$$
P_{k}(x)=: \sum_{r=0}^{k^{2}} c_{r}(k) x^{k^{2}-r} .
$$

In order to arrive at this conjecture, CFKRS considered a more general moment problem with 'shifts'. Because this general setting was central to our computation, we describe their conjecture with shifts below. Write the functional equation of zeta as

$$
\zeta(s)=\chi(s) \zeta(1-s)
$$

where

$$
\chi(s):=\pi^{s-1 / 2} \Gamma((1-s) / 2) / \Gamma(s / 2) .
$$

It is a little more convenient to work with the Hardy $Z$-function, whose functional equation and approximate functional equation are expressed more symmetrically than that of the zeta function. It is defined as

$$
Z(s)=\chi(s)^{-1 / 2} \zeta(s),
$$

and satisfies

$$
\begin{aligned}
& Z(s)=Z(1-s), \quad(\text { because } \chi(s) \chi(1-s)=1), \\
& Z(1 / 2+i t) \in \mathbb{R} \text { for } t \in \mathbb{R}, \\
& |Z(1 / 2+i t)|=|\zeta(1 / 2+i t)| .
\end{aligned}
$$

CFKRS took as their starting point the shifted moments:

$$
M\left(\alpha_{1}, \ldots, \alpha_{2 k}\right):=\int_{0}^{T} Z\left(1 / 2+i t+\alpha_{1}\right) \cdots Z\left(1 / 2+i t+\alpha_{2 k}\right) d t,
$$

where $\alpha_{j} \in \mathbb{C}$ are distinct and satisfy $-1 / 4<\Re \alpha_{j}$. When $\alpha=0$ the integrand is $|\zeta(1 / 2+i t)|^{2 k}$.

Substituting the approximate functional equation into each factor of the above integrand

$$
Z(s)=\chi(s)^{-1 / 2} \sum_{n \leq \sqrt{\frac{t}{2 \pi}}} \frac{1}{n^{s}}+\chi(1-s)^{-1 / 2} \sum_{n \leq \sqrt{\frac{t}{2 \pi}}} \frac{1}{n^{1-s}}+O\left(t^{-\sigma / 2}\right),
$$

$s=\sigma+i t, 0<\sigma<1$, CFKRS applied the following heuristic steps:

a) Ignore the $O\left(t^{-\sigma / 2}\right)$ and expand the product to get $2^{2 k}$ terms, each a product $2 k$ sums.

b) Of the $2^{2 k}$ terms, only the terms with the same number of $s$ 's and $1-s$ 's contribute to the asymptotics. Reasoning: $\chi(s)$ is highly oscillatory, so cancellation occurs unless each $s$ gets paired with a $1-s$.

c) For any such term, only the diagonal (' $m_{1} m_{2} \ldots m_{k}=n_{1} n_{2} \ldots n_{k}$ ') contributes when the sums are multiplied out. 
d) Extend the truncated diagonal sums to infinity, replacing the sums that diverge with their analytic continuation (the assumption we stated earlier, $-1 / 4<\Re \alpha_{j}$, is used when obtaining the analytic continuation of the diagonal sums).

The steps in this heuristic recipe are not justifiable, and individually are not even true! The terms that are dropped cannot be neglected, and it appears that some sort of cancellation takes place amongst these terms so that, in the end, the above steps do apparently result in a correct conjecture, as described below.

Let

$$
H\left(z_{1}, \ldots, z_{2 k} ; x\right):=\exp \left(\frac{x}{2} \sum_{1}^{k} z_{j}-z_{j+k}\right) G\left(z_{1}, \ldots, z_{2 k}\right) .
$$

The first conjectured asymptotic formula of CFKRS, which we refer to as the combinatorial sum, for shifted moments reads:

$$
M\left(\alpha_{1}, \ldots, \alpha_{2 k}\right) \sim \int_{0}^{T} P_{k}\left(\alpha, \log \frac{t}{2 \pi}\right) d t
$$

where

$$
P_{k}(\alpha, x)=\sum_{\sigma \in \Xi} H\left(\alpha_{\sigma(1)}, \ldots, \alpha_{\sigma(2 k)} ; x\right),
$$

and $\Xi \subset S_{2 k}$ is the set of $\left(\begin{array}{c}2 k \\ k\end{array}\right)$ permutations such that $\sigma(1)<\cdots<\sigma(k)$ and $\sigma(k+1)<\cdots<\sigma(2 k)$. The terms in this set correspond to the number of ways to select, from approximate functional equation for $Z(s)$, the same number $k$ of $s$ 's and $(1-s)$ 's.

CFKRS, also expressed their sum of $\left(\begin{array}{c}2 k \\ k\end{array}\right)$ terms as a $2 k$-fold residue. We reproduce their second formula for the shifted moments:

$$
\begin{gathered}
P_{k}(\alpha, x)=\frac{(-1)^{k}}{k !^{2}} \frac{1}{(2 \pi i)^{2 k}} \oint \cdots \oint \quad \frac{G\left(z_{1}, \ldots, z_{2 k}\right) \Delta\left(z_{1}, \ldots, z_{2 k}\right)^{2}}{\prod_{l=1}^{2 k} \prod_{j=1}^{2 k}\left(z_{l}-\alpha_{j}\right)} \\
\times e^{\frac{x}{2} \sum_{l=1}^{k} z_{l}-z_{l+k}} d z_{1} \ldots d z_{2 k} .
\end{gathered}
$$

The Vandermonde in the above residue vanishes whenever any of the $z_{j}$ 's are equal, thus restricting the residues to contributions from choosing a distinct $\alpha$ for each $z$, i.e., $z_{j}=\alpha_{\sigma(j)}$, where $\sigma$ is a permutation of $1,2, \ldots, 2 k$. The symmetry of the function $G$ with respect to the first $k$ variables, and with respect to the last $k$ variables allows one to write the resulting residues as a sum over the $\left(\begin{array}{c}2 k \\ k\end{array}\right)$ permutations $\Xi$ in (15). The residue computation establishing the equivalence of (16) and (15) is carried out in Lemma 2.5.3 of [CFKRS].

While the $\left(\begin{array}{c}2 k \\ k\end{array}\right)$ terms of the combinatorial sum (15) for $P_{k}(\alpha, x)$ each have poles of order $k^{2}$ at $\alpha=0$, the above is analytic in a neighbourhood of $\alpha=0$ which shows that these poles must cancel. Working with shifts allowed CFKRS to get beyond these poles.

To get formula (2) for $P_{k}(x)$, set $\alpha=0$ in (16). Even though the formula conjectured by CFKRS is complicated, it does seem to correctly predict the moments. Some evidence, both theoretical and numerical, in its favour was presented in CFKRS and CFKRS2. For instance, the formulas predicted by CFKRS match with known theorems, including lower terms, for $k=1,2$. For $k=1$, the full 
asymptotics were obtained by Ingham [I], when $\left|\alpha_{1}\right|,\left|\alpha_{2}\right|<1 / 2$. For $k=2$, the full asymptotics was proven by Heath-Brown [H-B] when $\alpha_{j}=0$, and Motohashi with shifts $\alpha_{j}$ in some neighbourhood of 0 .

Perhaps the best evidence for these heuristics is numerical. CFKRS computed the moment polynomials for $k \leq 7$, and tested the moment conjecture for $T$ roughly of size $10^{6}$. In this paper we test the conjecture for $k \leq 13$ and $T$ up to $10^{8}$. Additional numerics are discussed in $\mathrm{HO}$.

The heuristics do not shed much light on the nature of the remainder term. The related multiple Dirichlet series approach of Diaconu, Goldfeld, and Hoffstein [DGH] does predict, based on conjectured analytic properties of the relevant multiple Dirichlet series, a remainder term of size $O_{k, \varepsilon}\left(T^{1 / 2+\varepsilon}\right)$.

In CFKRS2, two methods are described for computing the coefficients $c_{r}(k)$ of $P_{k}(x)$. Their first method, based on deriving explicit formulas for the $c_{r}(k)$, taking equation (2) as the starting point, is useful for obtaining the coefficients to very high precision, but is limited to smaller values of $r$. In CFKRS] and CFKRS2, their first method was used to compute $c_{r}(k)$ for $r \leq 9$ and various values of $k$.

Their second method is more useful for computing all $k^{2}+1$ coefficients of $P_{k}(x)$, and is easier to implement, but is more limited in the amount of precision that it can achieve. It uses (15), small shifts $\alpha_{j}$, and high precision to capture cancellation resulting from high order poles that cancel in the terms of this sum.

In this paper we describe a 'cubic accelerant' variant of the second method presented in CFKRS2 for computing coefficients of the moment polynomials. This allowed us to extend tables of the $c_{r}(k)$ for $k \leq 13$, and, $0 \leq r \leq k^{2}$. We also computed many of the coefficients to greater precision. Finally, we used our tables of coefficients to test the moment conjectures up to $k \leq 13$ and $T$ up to $10^{8}$.

Going up to $k=13$, using (15), or more precisely using (25), is substantial, because computing the coefficients to $D$ digits accuracy involves evaluating $k^{2}$ sums (one for each $r$ ), each sum involving $\left(\begin{array}{c}2 k \\ k\end{array}\right)$ terms $(10,400,600$ for $k=13$ ), with working precision of roughly $D \times k^{2}$ digits of accuracy. For example, about 2000 digits are required for $k=13$ with desired precision of 12 digits. The process is made even more challenging by the fact that each term involves a complicated infinite multivariate product over primes. The computer data storage (RAM) requirements are also large because of the number of terms, $\left(\begin{array}{c}2 k \\ k\end{array}\right)$, that we are computing/updating one prime at a time to high precision.

The basic idea of the accelerant is to approximate the tail of the Euler product (5) defining $A_{k}$, with an expression, given in (35) that accounts for all terms of degree $\leq 3$ in $1 / p$. In our application, we will take the $z_{j}$ 's appearing in this approximation to be very small. This approximation gives, for large $p$, the local factor of $A_{k}$ up to a remainder of size roughly $O\left(k^{8} p^{-4}\right)$, so that the overall contribution, from all $p$ greater than a given large $P$, is approximated up to a remainder roughly of size $O\left(k^{8} P^{-3} \log (P)^{-1}\right)$. This is described in Section 4.2 .

For instance, the use of our cubic approximation (35) allowed us to truncate the multivariate Euler product $A_{13}$ at $P=1699$, and achieve the quality of coefficients described in Table 9 of Section 4 . Without this approximation, we would have needed to truncate at $P \approx 10^{9}$. For $k=4$, the results we obtained with $P=$ $942,939,827$ would have required $P \approx 10^{27}$ without any acceleration. 


\section{Computing the coefficients of $P_{k}(x)$}

The first method of CFKRS for computing the coefficients of $P_{k}(x)$ involved expanding, in a multivariate Taylor series, the integrand of (2), and working out a technique for expressing the resulting residue (for a general $k$ ), giving formulas for $c_{r}(k)$. For example,

$$
\begin{aligned}
c_{0}(k)= & a_{k} \prod_{j=0}^{k-1} \frac{j !}{(j+k) !} \\
c_{1}(k)= & c_{0}(k) 2 k^{2}\left(\gamma_{0} k+B_{k}(1 ;)\right) \\
c_{2}(k)= & c_{0}(k) k^{2}(k-1)(k+1) \\
& \times\left(2\left(B_{k}(1 ;)+\gamma_{0} k\right)^{2}-\gamma_{0}^{2}-2 \gamma_{1}+B_{k}(1,1 ;)-B_{k}(1 ; 1)\right),
\end{aligned}
$$

where

$$
a_{k}=\prod_{p}\left(1-p^{-1}\right)^{k^{2}}{ }_{2} F_{1}(k, k ; 1 ; 1 / p),
$$

the $\gamma_{j}$ 's defined by

$$
s \zeta(1+s)=1+\gamma_{0} s-\gamma_{1} s^{2}+\frac{\gamma_{2}}{2 !} s^{3}-\frac{\gamma_{3}}{3 !} s^{4}+\cdots,
$$

and

$$
\begin{aligned}
& B_{k}(1 ;)=\sum_{p} \frac{k \log (p)}{p-1}-\frac{\log (p) k_{2} F_{1}(k+1, k+1 ; 2 ; 1 / p)}{p_{2} F_{1}(k, k ; 1 ; 1 / p)} \\
& B_{k}(1,1 ;)=-\sum_{p}\left(\frac{\log (p)^{2} k_{2}^{2} F_{1}(k+1, k+1 ; 2 ; 1 / p)^{2}}{p^{2}{ }_{2} F_{1}(k, k ; 1 ; 1 / p)^{2}}\right. \\
& \left.-\frac{\log (p)^{2}\left(\begin{array}{c}
k+1 \\
2
\end{array}\right){ }_{2} F_{1}(k+2, k+2 ; 3 ; 1 / p)}{p^{2}{ }_{2} F_{1}(k, k ; 1 ; 1 / p)}\right) \\
& B_{k}(1 ; 1)=\sum_{p} \frac{p \log (p)^{2}}{(p-1)^{2}}+\left(\frac{\log (p)^{2} k^{2}{ }_{2} F_{1}(k+1, k+1 ; 2 ; 1 / p)^{2}}{p^{2}{ }_{2} F_{1}(k, k ; 1 ; 1 / p)^{2}}\right. \\
& \left.-\frac{\log (p)_{2}^{2} F_{1}(k+1, k+1 ; 1 ; 1 / p)}{p_{2} F_{1}(k, k ; 1 ; 1 / p)}\right) \\
& B_{k}(2 ;)=-\sum_{p} \frac{k p \log (p)^{2}}{(p-1)^{2}}+\left(\frac{\log (p)^{2} k^{2}{ }_{2} F_{1}(k+1, k+1 ; 2 ; 1 / p)^{2}}{p^{2}{ }_{2} F_{1}(k, k ; 1 ; 1 / p)^{2}}\right. \\
& -\frac{\log (p)^{2}\left(\begin{array}{c}
k+1 \\
2
\end{array}\right){ }_{2} F_{1}(k+2, k+2 ; 3 ; 1 / p)}{p^{2}{ }_{2} F_{1}(k, k ; 1 ; 1 / p)} \\
& \left.-\frac{\log (p)^{2} k_{2} F_{1}(k+2, k+1 ; 2 ; 1 / p)}{p_{2} F_{1}(k, k ; 1 ; 1 / p)}\right),
\end{aligned}
$$

with ${ }_{2} F_{1}$ Gauss's hypergeometric function.

These formulas quickly get much more complicated. In practice CFKRS were able to use this method for $r \leq 9$ and compute numerical approximations for all the coefficients of, for example, $P_{3}(x)$. One advantage of these formulas, expressed as sums over primes, is that one can apply Mobius inversion to accelerate the convergence of these sums, and obtain high precision values of the coefficients. 
It soon became apparent CFKRS from numerical values of $c_{r}(k)$ that the leadings coefficients of $P_{k}(x)$, i.e., associated to the larger powers of $x$, are very small in comparison to the lower terms. Thus, in order to meaningfully test the moment conjecture for zeta, which involves the moment polynomial evaluated at the slowly growing function $\log (t / 2 \pi)$ (and this hardly changes over the range of $t$ in which we can gather significant data for $\zeta(1 / 2+i t))$, one needs many coefficients of the moment polynomials. See also $[\mathrm{HR}]$ which discusses the uniform asymptotics of these coefficients.

Consequently, a second practical method, relying on the combinatorial sum (15), was developed for computing numerical approximations for all $k^{2}$ coefficients of the moment polynomial $P_{k}(x)$.

We detail our computational approach, implementation, and numerical results in the next two sections.

\section{Our numerical evaluation of $c_{r}(k)$}

The polynomial $P_{k}(x)$ given by (2) is the special case $\alpha_{1}=\ldots=\alpha_{2 k}=0$ of the function $P_{k}(\alpha, x)$ in (16). CFRKS's second method for computing the coefficients of $P_{k}(x)$ relies on their equation (15) for $P_{k}(\alpha, x)$. However, the terms in (15) have poles if the $\alpha_{j}$ 's are not distinct, coming from the product of $k^{2}$ zetas,

$$
\prod_{i=1}^{k} \prod_{j=1}^{k} \zeta\left(1+z_{i}-z_{j+k}\right)
$$

that appear in the function $G$. So we cannot simply substitute $\alpha_{j}=0$.

Instead we take the limit as $\alpha_{j} \rightarrow 0$ while making sure that all the $\alpha_{j}$ 's are distinct. Because of the poles, each individual term in (15) becomes very large when $\alpha$ is small, and high precision is needed to see one's way through the resulting cancellation of the poles as we sum across the $\left(\begin{array}{c}2 k \\ k\end{array}\right)$ terms of the combinatorial sum.

More precisely, consider

$$
H\left(z_{1}, \ldots, z_{2 k} ; x\right)=\exp \left(\frac{x}{2} \sum_{1}^{k} z_{j}-z_{j+k}\right) A_{k}\left(z_{1}, \ldots, z_{2 k}\right) \prod_{i=1}^{k} \prod_{j=1}^{k} \zeta\left(1+z_{i}-z_{j+k}\right),
$$

and let

$$
\delta_{j}=j \delta,
$$

where $\delta \in \mathbb{C}$ is a small number. In practice $\delta$ was of the form $10^{-D}$ for some positive integer $D$.

Using (15) we obtain

$$
P_{k}(x)=\lim _{\delta \rightarrow 0} \sum_{\sigma \in \Xi} H\left(\delta_{\sigma(1)}, \ldots, \delta_{\sigma(2 k)} ; x\right) .
$$

As in CFKRS2], we expand $\exp \left(\frac{x}{2} \sum_{1}^{k} z_{j}-z_{j+k}\right)$ in its Taylor series, and pull out the coefficient of $x^{k^{2}-r}$, to get

$$
c_{r}(k)=\frac{1}{2^{k^{2}-r}\left(k^{2}-r\right) !} \lim _{\delta \rightarrow 0} \sum_{\sigma \in \Xi} H_{r}\left(\delta_{\sigma(1)}, \ldots, \delta_{\sigma(2 k)}\right),
$$


where

$$
H_{r}\left(z_{1}, \ldots, z_{2 k}\right)=\left(\sum_{1}^{k} z_{j}-z_{j+k}\right)^{k^{2}-r} A_{k}\left(z_{1}, \ldots, z_{2 k}\right) \prod_{i=1}^{k} \prod_{j=1}^{k} \zeta\left(1+z_{i}-z_{j+k}\right) .
$$

Notice that, as a function of $\delta, H_{r}\left(\delta_{\sigma(1)}, \ldots, \delta_{\sigma(2 k)}\right)$ has a pole at $\delta=0$ of order $r$, because the first factor above cancels $k^{2}-r$ of the $k^{2}$ poles of the double product of zetas. These poles must cancel when summed over permutations $\sigma$, otherwise we would not obtain the lhs, $c_{r}(k)$, as $\delta \rightarrow 0$. Therefore, because the sum in (25) is analytic about $\delta=0$, we may write

$$
c_{r}(k)=\frac{1}{2^{k^{2}-r}\left(k^{2}-r\right) !}\left(\left(\sum_{\sigma \in \Xi} H_{r}\left(\delta_{\sigma(1)}, \ldots, \delta_{\sigma(2 k)}\right)\right)+O(|\delta|)\right),
$$

with the implied constant in the remainder term depending on $k$ and $r$. In our implementation, we neglected the contribution from the $O(|\delta|)$ term for reasons that are described at the end of subsection 4.2 .

One complication in evaluating the above for a given $k$ and $\delta$ is that $A_{k}\left(z_{1}, \ldots, z_{2 k}\right)$ is expressed as an infinite product over primes as described by (5).

While CFKRS used a 'quadratic accelerant' for evaluating the multivariate Euler product, we implemented a cubic accelerant. This has the advantage of allowing us to truncate the Euler product sooner.

To evaluate the convergent product $A_{k}\left(z_{1}, \ldots, z_{2 k}\right)$, we break up the product over primes into $p \leq P$ and $p>P$, where $P$ is a large number. For the first portion $p \leq P$, we use the following identity, derived in Section 2.6 of [CFKRS,

$$
A_{k}\left(z_{1}, \ldots, z_{2 k}\right)=\prod_{p} \sum_{j=1}^{k} \prod_{i \neq j} \frac{\prod_{m=1}^{k}\left(1-p^{-1+z_{i+k}-z_{m}}\right)}{1-p^{z_{i+k}-z_{j+k}}},
$$

to numerically compute the local factor of $A_{k}\left(z_{1}, \ldots, z_{2 k}\right)$ for specific values of $p$ and $z_{1}, \ldots, z_{2 k}$. Ideally, we would compute the local factor for all $p$ using this formula, but, because there are infinitely many primes, we must eventually stop. However, we will describe a method to approximate the contribution from all $p>P$, thus allowing us to attain higher precision in our computation with fewer primes. The choice of $P$ is described in subsection 4.2 .

Some care must be taken to account for the fact that individual terms in (28) also have poles. While these poles cancel out when summed over $j$ (see the paragraph following equation (2.6.16) in CFKRS]), they cause some additional loss of precision in our application. We are evaluating $A_{k}\left(z_{1}, \ldots, z_{2 k}\right)$ at distinct, but small values of $z_{j}$. Therefore, when evaluating the sum over $j$, additional cancellation and hence loss of precision occurs, affecting the leading $(k-1) D$ digits of the truncated Euler product for $A_{k}\left(z_{1}, \ldots, z_{2 k}\right)$, where $z_{j} \approx 10^{-D}$, from the poles of order $k-1$ of the individual terms summed in (28).

For the contribution of the second portion $p>P$, we approximate each local factor appearing in (5) by a product of zeta functions that captures the terms up to degree three in $1 / p$ of its multivariate Dirichlet series. A cubic approximation can be obtained by first substituting $u_{j}=p^{-1 / 2-z_{j}}$ and $w_{j}=p^{-1 / 2+z_{k+j}}$ into the 
local factor of (5),

$$
\prod_{l, j=1}^{k}\left(1-u_{l} w_{j}\right) \int_{0}^{1} \prod_{j=1}^{k}\left(1-u_{j} e(\theta)\right)^{-1}\left(1-w_{j} e(-\theta)\right)^{-1} d \theta
$$

and then working out the terms, in the multivariate Maclaurin series, up to degree six, in $u_{j}$ and $w_{j}$.

Notice that the integral over $\theta$ pulls out just the terms with the same number of $u$ 's and $w$ 's. This results in monomials only of even degree appearing. The integral of any other term, which does not have the same number of $u$ 's and $w$ 's, is zero because it contains a non-zero integer power of $e(\theta)$.

Also, observe that the local factor of (29) is symmetric in the $u$ 's and, separately in the $w$ 's, meaning if the $u_{l}$ 's are permuted the expression remains invariant and similarly for the $w_{l}$ 's. Also, it is symmetric with $u$ and $w$, i.e., if all the $u$ 's and $w$ 's are swapped the expression remains the same.

Therefore, to get terms up to degree six, we can determine the coefficients of representative terms involving $u_{1}, u_{2}, u_{3}$ and $w_{1}, w_{2}, w_{3}$, and then symmetrize the resulting expressions over all the $u$ 's and $w$ 's. More precisely, to get all terms of degrees 2,4 , and 6 , it is sufficient to consider only the monomials: $u_{1} w_{1}, u_{1} u_{2} w_{1} w_{2}$, $u_{1} u_{2} w_{1}^{2}, u_{1}^{2} w_{1}^{2}, u_{1} u_{2} u_{3} w_{1} w_{2} w_{3}, u_{1} u_{2} u_{3} w_{1}^{2} w_{2}, u_{1} u_{2} u_{3} w_{1}^{3}, u_{1}^{2} u_{2} w_{1}^{2} w_{2}, u_{1}^{2} u_{2} w_{1}^{3}$, and $u_{1}^{3} w_{1}^{3}$ instead of every possible monomial, and then exploit symmetry.

Finally, we can simplify further. The integral over $\theta$ simply plays the role of pulling out terms with the same number of $u$ 's and $w$ 's. So, instead of (29), we can work more directly with the function

$$
\prod_{l, j=1}^{k}\left(1-u_{l} w_{j}\right) \prod_{j=1}^{k}\left(1-u_{j}\right)^{-1}\left(1-w_{j}\right)^{-1} .
$$

The multivariate Maclaurin series of the above coincides with that of (29) for those terms that have the same number of $u$ 's and w's. Furthermore, because we are focusing just on terms involving $u_{1}, u_{2}, u_{3}$ and $w_{1}, w_{2}, w_{3}$, we can set $u_{j}=w_{j}=0$ for all $j \geq 4$. Finally, to get terms up to degree six with the same number of $u$ 's and $w$ 's, we can expand each factor in the denominator as a geometric series of degree 3. We therefore consider:

$$
\prod_{l, j=1}^{3}\left(1-u_{l} w_{j}\right) \prod_{j=1}^{3}\left(1+u_{j}+u_{j}^{2}+u_{j}^{3}\right)\left(1+w_{j}+w_{j}^{2}+w_{j}^{3}\right) .
$$

We expand out the above and tabulate, in Table1 the coefficients for representative monomials with the same number of $u$ 's and $w$ 's, up to degree 6 , in the multivariate Maclaurin series of the above function, and, hence, equivalently, in (29).

Therefore, symmetrizing, we have that (29) equals

$$
\begin{array}{r}
1-\sum_{\substack{1 \leq i_{1}<i_{2} \leq k \\
1 \leq j_{1}<j_{2} \leq k}} u_{i_{1}} u_{i_{2}} w_{j_{1}} w_{j_{2}}+4 \sum_{\substack{1 \leq i_{1}<i_{2}<i_{3} \leq k \\
1 \leq j_{1}<j_{2}<j_{3} \leq k}} u_{i_{1}} u_{i_{2}} u_{i_{3}} w_{j_{1}} w_{j_{2}} w_{j_{3}} \\
(32)+\sum_{\substack{1 \leq i_{1}<i_{2}<i_{3} \leq k \\
1 \leq j_{1} \neq j_{2} \leq k}} u_{i_{1}} u_{i_{2}} u_{i_{3}} w_{j_{1}}^{2} w_{j_{2}}+\sum_{\substack{1 \leq i_{1} \neq i_{2} \leq k \\
1 \leq j_{1}<j_{2}<j_{3} \leq k}} u_{i_{1}}^{2} u_{i_{2}} w_{j_{1}} w_{j_{2}} w_{j_{3}}+\ldots .
\end{array}
$$


TABLE 1. The second column lists the coefficients that appear with representative monomials, up to degree 6 , in the multivariate Maclaurin expansion of (29).

\begin{tabular}{|c|c|}
\hline monomial & coefficient \\
\hline$u_{1} w_{1}$ & 0 \\
$u_{1} u_{2} w_{1} w_{2}$ & -1 \\
$u_{1} u_{2} w_{1}^{2}$ & 0 \\
$u_{1}^{2} w_{1}^{2}$ & 0 \\
$u_{1} u_{2} u_{3} w_{1} w_{2} w_{3}$ & 4 \\
$u_{1} u_{2} u_{3} w_{1}^{2} w_{2}$ & 1 \\
$u_{1} u_{2} u_{3} w_{1}^{3}$ & 0 \\
$u_{1}^{2} u_{2} w_{1}^{2} w_{2}$ & 0 \\
$u_{1}^{2} u_{2} w_{1}^{3}$ & 0 \\
$u_{1}^{3} w_{1}^{3}$ & 0 \\
\hline
\end{tabular}

Undoing the substitution for $u_{l}$ 's and $w_{j}$ 's, gives the following expansion for the local factors in (5):

$$
\begin{gathered}
1-\sum_{\substack{1 \leq i_{1}<i_{2} \leq k \\
1 \leq j_{1}<j_{2} \leq k}} p^{-2-z_{i_{1}}-z_{i_{2}}+z_{k+j_{1}}+z_{k+j_{2}}} \\
+4 \sum_{\substack{1 \leq i_{1}<i_{2}<i_{3} \leq k \\
1 \leq j_{1}<j_{2}<j_{3} \leq k}} p^{-3-z_{i_{1}}-z_{i_{2}}-z_{i_{3}}+z_{k+j_{1}}+z_{k+j_{2}}+z_{k+j_{3}}} \\
+\sum_{\substack{1 \leq i_{1}<i_{2}<i_{3} \leq k \\
1 \leq j_{1} \neq j_{2} \leq k}} p^{-3-z_{i_{1}}-z_{i_{2}}-z_{i_{3}}+2 z_{k+j_{1}}+z_{k+j_{2}}} \\
+\sum_{\substack{1 \leq i_{1} \neq i_{2} \leq k \\
1 \leq j_{1}<j_{2}<j_{3} \leq k \\
+\ldots,}} p^{-3-2 z_{i_{1}}-z_{i_{2}}+z_{k+j_{1}}+z_{k+j_{2}}+z_{k+j_{3}}} \\
+\ldots
\end{gathered}
$$

which we then approximate by the following product:

$$
\begin{aligned}
& \prod_{\substack{1 \leq i_{1}<i_{2} \leq k \\
1 \leq j_{1}<j_{2} \leq k \\
\times}}\left(1-p^{\left.-2-z_{i_{1}}-z_{i_{2}}+z_{k+j_{1}}+z_{k+j_{2}}\right)}\right. \prod_{\substack{1 \leq i_{1}<i_{2}<i_{3} \leq k \\
1 \leq j_{1}<j_{2}<j_{3} \leq k}}\left(1-p^{-3-z_{i_{1}}-z_{i_{2}}-z_{i_{3}}+z_{k+j_{1}}+z_{k+j_{2}}+z_{k+j_{3}}}\right)^{-4} \\
& \times \prod_{\substack{1 \leq i_{1}<2_{2}<i_{3} \leq k \\
1 \leq j_{1} \neq j_{2} \leq k}}\left(1-p^{-3-z_{i_{1}}-z_{i_{2}}-z_{i_{3}}+2 z_{k+j_{1}}+z_{k+j_{2}}}\right)^{-1} \\
& \quad \prod_{\substack{1 \leq i_{1} \neq i_{2} \leq k \\
1 \leq j_{1}<j_{2}<j_{3} \leq k}}\left(1-p^{-3-2 z_{i_{1}}-z_{i_{2}}+z_{k+j_{1}}+z_{k+j_{2}}+z_{k+j_{3}}}\right)^{-1} .
\end{aligned}
$$

The last step can be seen by expanding each factor in a geometric series and comparing the terms, up to those containing a $1 / p^{3}$, with those in (33). We also remark that, had we wanted a quartic approximation, then slightly more care would be 
needed as the first product above would, on expanding in geometric series, interact with the quartic terms.

The product in (34) allows us to approximate the tail, i.e., for $p>P$, of $A_{k}\left(z_{1}, \ldots, z_{k}\right)$ in terms of the Riemann zeta function:

$$
\begin{gathered}
\prod_{p>P} \prod_{l, j=1}^{k}\left(1-p^{-1-z_{l}+z_{k+j}}\right) \int_{0}^{1} \prod_{j=1}^{k}\left(1-\frac{e(\theta)}{p^{\frac{1}{2}+z_{j}}}\right)^{-1}\left(1-\frac{e(-\theta)}{p^{\frac{1}{2}-z_{k+j}}}\right)^{-1} d \theta \\
\approx \frac{\prod_{\substack{1 \leq i_{1}<i_{2} \leq k \\
1 \leq j_{1}<j_{2} \leq k}} \zeta\left(2+z_{i_{1}}+z_{i_{2}}-z_{k+j_{1}}-z_{k+j_{2}}\right)^{-1}}{\prod_{p \leq P} \prod_{\substack{1 \leq i_{1}<i_{2} \leq k \\
1 \leq j_{1}<j_{2} \leq k}}\left(1-p^{\left.-2-z_{i_{1}}-z_{i_{2}}+z_{k+j_{1}}+z_{k+j_{2}}\right)}\right.} \\
\quad \times \frac{\prod_{\substack{1 \leq i_{1}<i_{2}<i_{3} \leq k \\
1 \leq j_{1}<j_{2}<j_{3} \leq k}} \zeta\left(3+z_{i_{1}}+z_{i_{2}}+z_{i_{3}}-z_{k+j_{1}}-z_{k+j_{2}}-z_{k+j_{3}}\right)^{4}}{\prod_{p \leq P} \prod_{\substack{1 \leq i_{1}<i_{2}<i_{3} \leq k \\
1 \leq j_{1}<j_{2}<j_{3} \leq k}}\left(1-p^{\left.-3-z_{i_{1}}-z_{i_{2}}-z_{i_{3}}+z_{k+j_{1}}+z_{k+j_{2}}+z_{k+j_{3}}\right)^{-4}}\right.} \\
\times \frac{\prod_{\substack{1 \leq i_{1}<i_{2}<i_{3} \leq k \\
1 \leq j_{1} \neq j_{2} \leq k}} \zeta\left(3+z_{i_{1}}+z_{i_{2}}+z_{i_{3}}-2 z_{k+j_{1}}-z_{k+j_{2}}\right)}{\prod_{p \leq P} \prod_{\substack{1 \leq i_{1}<i_{2}<i_{3} \leq k \\
1 \leq j_{1} \neq j_{2} \leq k}}\left(1-p^{\left.-3-z_{i_{1}}-z_{i_{2}}-z_{i_{3}}+2 z_{k+j_{1}}+z_{k+j_{2}}\right)^{-1}}\right.} \\
\times \frac{\prod_{\substack{1 \leq i_{1} \neq i_{2} \leq k \\
1 \leq j_{1}<j_{2}<j_{3} \leq k}} \zeta\left(3+2 z_{i_{1}}+z_{i_{2}}-z_{k+j_{1}}-z_{k+j_{2}}-z_{k+j_{3}}\right)}{\prod_{p \leq P} \prod_{\substack{1 \leq i_{1} \neq i_{2} \leq k \\
1 \leq j_{1}<j_{2}<j_{3} \leq k}}\left(1-p^{\left.-3-2 z_{i_{1}}-z_{i_{2}}+z_{k+j_{1}}+z_{k+j_{2}}+z_{k+j_{3}}\right)^{-1}}\right.} .
\end{gathered}
$$

\section{Implementation AND tables of COEFficients $c_{r}(k)$}

Our code was implemented in C++ using the GNU MPFR library FHLPZ, along with Jon Wilkening's C++ wrapper for MPFR. MPFR is based on GMP, the GNU multiprecision library. We used gcc, the GNU C compiler, to compile our code, with the '-fopenmp' option in order to enable the use of OpenMP directives in our code. This allowed us to carry out some of the key steps in parallel for a given $k$, using several cores of our machine. Computations were carried out on an SGI Altix 3700 computer with 64 Itanium2 processors and 192 GB of shared memory.

For each $k$, we selected a precision, specified by the number of digits desired, 'Digits', for the final output, and let $\delta=10^{- \text {Digits }}$. For example, we used Digits $=25$, i.e., $\delta=10^{-25}$, for $k=4$. We then put $\delta_{j}=j \delta, 1 \leq j \leq k$, and set about computing the sum (27), using our cubic multivariate approximation, i.e., equation (35), for the tail of the infinite product $A_{k}\left(z_{1}, \ldots, z_{2 k}\right)$.

Observe, in (26), that the dependence on $r$ manifests only at the factor:

$$
\left(\sum_{1}^{k} z_{j}-z_{j+k}\right)^{k^{2}-r}
$$

Therefore, we were able to store and recycle all the other quantities across $0 \leq r \leq$ $k^{2}$.

We record one important hack that we used several times in our program. While the double product of zetas in (26) involves $k^{2}$ factors, many of these are repeated since there are just $4 k-2$ possible values of $\delta_{a}-\delta_{b}=(a-b) \delta$, where $a, b$ are distinct integers in $[1,2 k]$. The same holds, for each $p$, in the double products in (28).

Likewise, while the products in (35) involve up to $O\left(k^{6}\right)$ factors, these appear with multiplicity, and there are just $O(k)$ distinct factors. This is true both for the 
product of zetas in the numerator, and also, for each $p$, the factors that appear in the denominator.

We exploited these multiplicities by computing and storing a table of the distinct values of zeta that appear and, for each $p$, of the distinct factors that occur. Furthermore, we took advantage of the fact that the powers of $p$ that occur, other than $1 / p$, are of the form $p^{m \delta}$, where $m \in \mathbb{Z}$, and thus computed and stored them by repeated multiplication of $p^{\delta}$ and of $1 / p^{\delta}$.

This allowed us to avoid recomputing the same quantities repeatedly and also to cut back significantly on the amount of high precision multiplications needed. For instance, we looped through the various indices in (35) to count which factors appear with which multiplicities. This could be done simply, for our range of $k$, using 32 bit integer arithmetic, with $O(k)$ exponentiations and multiplications then carried out for each $p$ in (35), rather than $O\left(k^{6}\right)$ multiplications.

To account for the high amount of cancellation that occurs as a consequence of the poles of the individual terms in (27), we let our working precision be equal to

$$
\text { WorkingDigits }=\left(k^{2}+k-1+6\right) \text { Digits, }
$$

and carried out our computations using these many digits. The $k^{2}$ was to account for the largest order poles occurring in $H_{r}$, when $r=k^{2}$, of order $k^{2}$. While we could have gotten away with less precision for smaller $r$, we recycled most of the computed quantities across all $r$. The $k-1$ accounts for cancellation amongst the poles of the terms in the sum over $j$ in (28). Finally, we needed to have some working precision left over, after all the cancellation, to capture $c_{r}(k)$ to Digits precision. The +6 Digits was chosen to give us some leeway. For example, we had WorkingDigits $=625$ for $k=4$ and Digits $=25$, and WorkingDigits $=2244$ for $k=13$ and Digits $=12$.

While it would have been preferable to use a larger value of Digits for all our $k$, rather than, for instance, a smaller value of Digits for $k=13$, two things made this prohibitive.

The first was computing time. For $k=13$, our program ran on 6 processors (using OpenMP to parallelize our code) for around 6 months, representing about $3 \mathrm{CPU}$ years. Setting Digits $=25$ for $k=13$, say, and thus WorkingDigits of around 4000 rather than 2000, would have at least doubled the amount of computing time needed to carry out each arithmetic operation, as explained in the next two subsections. Furthermore, to see the benefit of using a smaller value of $\delta=10^{-25}$ for $k=13$ would have required us to truncate our product over primes at a much larger $P$, around $10^{8}$ or $10^{9}$, as explained below, rather than $P=1699$ that we achieved for $k=13$, thus requiring roughly $10^{5}$ to $10^{6} \mathrm{CPU}$ years.

For smaller values of $k$, because of the lower complexity, we were able to achieve much larger values of $P$, and thus it made sense to set Digits larger. For example we achieved $P=942,939,827$ for $k=4$ with Digits $=25$, and $P=1,212,569$ for $k=8$ with Digits $=16$. Our specific choice of Digits and $P$ is listed for each $k$ in the captions of Tables 29 .

Second, the machine that we used is a multi-user machine and, even though it has 192 GB RAM, we were running processes for several $k \leq 13$ simultaneously and also competing for the machine's resources with other researchers and projects, so we had to temper our use of the machine's memory and processors. 
Note that the memory requirements are substantial, using

$$
O\left(\left(\begin{array}{c}
2 k \\
k
\end{array}\right) k^{2} \text { Digits }\right)
$$

bits for the storage as we loop through $p$ to compute the terms of the terms in (25).

4.1. Complexity analysis. A rough estimate of the complexity involved in our computations can now be described. To numerically compute each $c_{r}$, with $0 \leq$ $r \leq k^{2}$, we needed to sum the $\left(\begin{array}{c}2 k \\ k\end{array}\right)$ terms of (25). Notice that the dependence on $r$ only appears in the first factor of $H_{r}$ in (26).

The bulk of our computing time was spent in evaluating the $\left(\begin{array}{c}2 k \\ k\end{array}\right)$ Euler products $A_{k}\left(\delta_{\sigma(1)}, \ldots, \delta_{\sigma(2 k)}\right)$, and we limit ourselves to describing the complexity of computing the local factors, for all $p \leq P$, in (28), and the cubic accelerant (35), to $\approx k^{2}$ Digits decimal places. The choice of $P$ will be described in the next subsection.

To compute the local factor at $p$ expressed in (28) requires, to begin with, one exponentiation, namely $p^{\delta}$, one division to get $p^{-\delta}$, and $O(k)$ multiplications to compute all relevant powers of $p^{\delta}$ which we then store. In fact, these values can be used, for given $p$, across all $\left(\begin{array}{c}2 k \\ k\end{array}\right)$ terms of (25), and thus form an insignificant portion of the overall computation.

We can rewrite the local factor of (28) as

$$
\begin{aligned}
& \left(\prod_{i=1}^{k} \prod_{m=1}^{k}\left(1-p^{-1+z_{i+k}-z_{m}}\right)\right) \\
& \quad \times \sum_{j=1}^{k}\left(\prod_{i \neq j}\left(1-p^{z_{i+k}-z_{j+k}}\right)\right)^{-1}\left(\prod_{m=1}^{k}\left(1-p^{-1+z_{j+k}-z_{m}}\right)\right)^{-1} .
\end{aligned}
$$

In our application, we set $z_{j}=\sigma(j) \delta$, with $\sigma \in \Xi$.

The double product outside the sum can be evaluated using our table of values of powers of $p^{\delta}$, and $O\left(k^{2}\right)$ multiplications. The number of multiplications can be reduced to $O(k \log k)$ if we loop through the indices to count multiplicities amongst the terms that appear, and use repeated squaring, say, to carry out the powers of each factor (when counted according to multiplicity). Recall that there are only $O(k)$ possible values for the factors in the product.

Each term of the above sum over $j$ can be computed using $O(k)$ multiplications and one division. For each $p$, we also precomputed, stored, and recycled the possible values, of which there are $O(k)$, of the individual factors that appear in the sum, as they occur frequently over the $\left(\begin{array}{c}2 k \\ k\end{array}\right)$ terms in (25). Thus, the sum over $j$ involves, altogether, $O\left(k^{2}\right)$ multiplications of precomputed values. The precomputed values were then discarded once we moved onto the next $p$.

Hence, evaluating (28) for all $p \leq P$ can be achieved using

$$
\ll \frac{P k^{2}}{\log P}
$$

arithmetic operations (mainly multiplications) on numbers of $O\left(k^{2}\right.$ Digits) bits. The factor $P / \log P$ is to account, asymptotically, for the number of primes up to $P$. Using a Fast Fourier Transform method (such as Schönhage-Strassen's algorithm) for the high precision multiplications, the contribution from computing the local 
factors of (28) for all $p \leq P$ across all $\left(\begin{array}{c}2 k \\ k\end{array}\right)$ terms of (25) involves, for any $\epsilon>0$,

$$
\begin{aligned}
& \ll\left(\begin{array}{c}
2 k \\
k
\end{array}\right) k^{4+\epsilon} \operatorname{Digits}^{1+\epsilon} \frac{P}{\log P}, \\
& \ll 2^{2 k} k^{7 / 2+\epsilon} \operatorname{Digits}^{1+\epsilon} \frac{P}{\log P}
\end{aligned}
$$

bit operations, the latter bound following from Stirling's formula applied to the binomial coefficient.

The evaluation of the rhs of (35) can be carried out using similar methods. The bulk of the computation is spent in evaluating the product over $p \leq P$ that appears in the denominator. While the product involves $O\left(k^{6}\right)$ factors (in the product over $\left.i_{1}, i_{2}, i_{3}, j_{1}, j_{2}, j_{3}\right)$ there are, for each $p$, just $O(k)$ possible values for each factor when $z_{j}=\sigma(j) \delta, 1 \leq \sigma(j) \leq 2 k$. Hence these factors appear with high multiplicity. Furthermore, the multiplicities are independent of $p$, and we can count these and store the multiplicities for each of the $\left(\begin{array}{c}2 k \\ k\end{array}\right)$ permutations $\sigma$. The bit complexity in counting these powers is

$$
\ll\left(\begin{array}{c}
2 k \\
k
\end{array}\right) k^{6+\epsilon}
$$

Looking ahead to our overall bit complexity in (50), we find that counting powers is a small portion of the overall time needed.

Hence, by counting these multiplicities and using repeated squaring, we can compute the denominator of (35) with $O(k \log k)$, rather than $O\left(k^{6}\right)$, high precision multiplications. Thus, the complexity in computing (35), for given $P$, is dominated by (41).

4.2. Choice of $P$. We now give an estimate, with an explicit dependence on $k$, in the error in $A_{k}$ from truncating its Euler product (5) at given $P$. The local factor at $p$ of $A_{k}\left(\delta_{\sigma(1)}, \ldots, \delta_{\sigma(2 k)}\right)$ can be expanded in a series whose terms are of the form $1 / p^{r+m \delta}$, where $r \geq 0$ and $-2 r k \delta \leq m \leq 2 r k \delta$ (the latter because $\delta_{j}=j \delta$ with $1 \leq j \leq 2 k)$.

These arise in (29) by matching up an equal number of $e(\theta)$ 's with $e(-\theta)$ 's in the integrand of that formula. In the same way that we worked out the terms up to degree six in (29), we may consider the terms of degree $2 r$ by passing to (30), and focusing on the terms that have the same number, $r$, of $u$ 's and $w$ 's.

We can get an upper bound on the sum total of the terms of degree $2 r$ in (29) by examining the coefficient of $x^{2 r}$ in

$$
\left(1+x^{2}\right)^{k^{2}}(1-x)^{-2 k}
$$

with $x=p^{-1 / 2+2 k \delta}$. To get this expression, we have replaced the minus sign in (30) with a plus sign, so that both $\left(1+x^{2}\right)^{k^{2}}$ and $(1-x)^{-2 k}$ have Macluarin series with positive coefficients, and all the $u_{j}$ 's and $w_{j}$ 's with $x$ 's. 
Now, expanding $\left(1+x^{2}\right)^{k^{2}}$ and $(1-x)^{-2 k}$ in their Maclaurin series, and multiplying out gives

$$
\left(1+x^{2}\right)^{k^{2}}(1-x)^{-2 k}=\sum_{0}^{\infty} h_{m}(k) x^{m},
$$

with

$$
h_{m}(k)=\sum_{0 \leq j \leq m / 2}\left(\begin{array}{c}
k^{2} \\
j
\end{array}\right) \frac{2 k(2 k+1) \ldots(2 k+m-2 j-1)}{(m-2 j) !} .
$$

Now, because $(2 k+l) / l \leq k+1$ if $l \geq 2$, and because $\sum 1 / j$ ! converges, we have

$$
\left|h_{m}(k)\right| \ll(k+1)^{m} .
$$

Our application of this bound is to the case $m=2 r$.

In a similar way, we can bound the terms involving $p^{-r}$ on expanding out the products in (34), and prove that

$$
\begin{aligned}
& \prod_{p} \prod_{l, j=1}^{k}\left(1-p^{-1-z_{l}+z_{k+j}}\right) \int_{0}^{1} \prod_{j=1}^{k}\left(1-\frac{e(\theta)}{p^{\frac{1}{2}+z_{j}}}\right)^{-1}\left(1-\frac{e(-\theta)}{p^{\frac{1}{2}-z_{k+j}}}\right)^{-1} d \theta \\
& =(\text { equation (34) })\left(1+O\left(k^{8} p^{-4+8 k \delta}\right)\right) .
\end{aligned}
$$

Hence, the relative error in using (35) to approximate $A_{k}\left(\delta_{\sigma(1)}, \ldots, \delta_{\sigma(2 k)}\right)$ is

$$
\ll k^{8} \sum_{p>P} p^{-4+8 k \delta} \ll k^{8} \log (P)^{-1} P^{-3+8 k \delta} /(3-8 k \delta),
$$

assuming that $3>8 k \delta$. Recall that $\delta=10^{\text {Digits }}$. Thus, for

$$
P \gg k^{8 / 3} 10^{\text {Digits/3 }} \text {, }
$$

and also assuming that Digits satisfies, say, $k(\log (k)+$ Digits $) 10^{- \text {Digits }}<1 / 100$ (this easily satisfied condition allows us to ignore the $8 k \delta$ ), we have that the relative error in $A_{k}\left(\delta_{\sigma(1)}, \ldots, \delta_{\sigma(2 k)}\right)$ from truncating the Euler product at $P$ is $<10^{- \text {Digits }}$.

Without using (35) to accelerate the convergence of the Euler product, we would need $P \approx k^{4} 10^{\text {Digits }}$ to achieve comparable accuracy, and using a degree 2 approximation, rather than degree 3 , would require $P \approx k^{3} 10^{\text {Digits } / 2}$. The powers on $k$ here arise as in (47).

Combining (41) with this choice of $P$ gives a bound for the number of bit operations used:

$$
\ll 2^{2 k} k^{37 / 6+\epsilon} \operatorname{Digits}^{\epsilon} 10^{\text {Digits/3 }} \text {. }
$$

Note that using a specific desired precision, Digits, does not necessarily result in that precision being achieved as one also needs to take into account the implied constant in the $O$ term in (27) which depends both on $k$ and on $r$. Ideally, in place of this $O$-term, one would like to have uniform bounds with the dependence on $k$ and $r$ explicitly given. However, this is daunting for several reasons. First, the terms being summed in (27) have poles, with respect to $\delta$, of order $r$, that cancel, with the most challenging case being when $r=k^{2}$. To see one's way through this enormous cancellation involves examining the terms of the multi-variate series expansions of the summand up to degree $r$. In fact, our algorithm circumvents this 
difficulty (experimentally) by using high precision to account for the high amount of cancellation resulting from the high order poles that annihilate one another.

One could give extremely crude estimates for this $O$-term by using the $2 k$-fold residue that results from taking, in (16), the $r$-th term on expanding the exp in its Maclaurin series (with respect to the $x$ variable), and then differentiating, with respect to the shifts, under the integral sign.

The $2 k$-fold residue has the advantage of being analytic when the shifts $\alpha_{j}$ are set to 0 , i.e., it encodes the cancelling of the poles in (27). However, this advantage is deceptive, as the high multiplicity of the factors in the denominator of (16) introduces terms of high degree into the analysis. Furthermore, the $c_{r}(k)$ 's are, for the large part, very small, as can be seen in our tables. Estimates on the $c_{r}(k)$ 's alone have only been successfully carried out for a relatively small range of $r$, specifically, $r<k^{\beta}$, where $\beta<1[\mathrm{HR}$. Presumably the implied constants in the $O$-term are also comparatively small, and crudely bounding the integrand of the $2 k$-fold residue will thus not produce estimates useful in asserting the numerical correctness of the coefficients in our tables.

In practice, rather than working with explicit constants in the truncation bounds, both for the $O$ term in (27) and the Euler product, we experimented by taking different values of $\delta=10^{- \text {Digits }}$ and $P$, using our estimates as guides. We inspected the stability of our numerical values of $c_{r}(k)$ by comparing those computed for a given $P$ against those with $P$ replaced by the first prime smaller than $P / 3$, and only outputting the digits that agreed. It seems, from our tables, that the coefficients $c_{r}(k)$ with mid-range values of $r$ are more stable and converge faster with respect to $P$, especially for larger values of $k$. We did not explore the reason for this, but presumably the lower terms, beyond those resulting from our cubic accelerant, have comparatively smaller coefficients for those values of $r$ for which $c_{r}(k)$ converges faster.

Numerical values of the coefficients for $4 \leq k \leq 13$ thus obtained are presented in the Tables 29 below in scientific ' $\mathrm{e}$ ' notation; for example, $1.2 e-3=1.2 \times 10^{-3}$. High precision values of $c_{r}(k)$ for $k=1,2,3$, can be obtained from [CFKRS].

It is also worth mentioning that all the digits of the coefficients computed in this manner agree, except in a few instances where the last decimal place differs slightly, with the results of the first method of CFKRS2 (see Section 2). That method has the advantage of producing high precision values of the coefficients, but is limited to relatively small values of $r$. We reran the program used in CFKRS2 for $k \leq 13$ and $r \leq 7$ and display those values in Table 10 for comparison.

\section{Checking the moment polynomial Conjectures}

Let

$$
\operatorname{Data}_{k}(T)=\int_{T_{0}}^{\mathrm{T}}|\zeta(1 / 2+i t)|^{2 k} d t,
$$

with $T_{0}=14.134725 \ldots$ being the imaginary part of the first non-trivial zero of zeta, and let

$$
\operatorname{Conjecture~}_{k}(T)=\int_{T_{0}}^{\mathrm{T}} P_{k}\left(\log \frac{t}{2 \pi}\right) d t \text {. }
$$


We used our tables of $c_{r}(k)$ and a simple integration by parts to compute the latter for given $T$. Note that because the leading coefficients of $P_{k}(x)$ are very small, in the range of $T$ considered, the $c_{r}(k)$ 's with midrange and higher values of $r$ contribute the dominant amount to the integral. To accurately compute the prediction of [CFKRS] one does need the lower terms of the polynomial $P_{k}(x)$. Table 11 displays, for $1 \leq k \leq 13$ and $T=10^{8}$, the $r$ for which the corresponding term in (52) contributes the dominant amount to integral.

For example, when $k=3$ and $T=10^{8}$, the $r=2$ term of $P_{3}$ contributes the most to (52). Furthermore, the first four digits of (52), for $k=3$ and $T=10^{8}$, arise from all of the terms with $0 \leq r \leq 8$, while the $r=9$ term contributes to around the 6 th decimal place. Thus, the impact of omitting any of the terms $0 \leq r \leq 8$ would be readily seen in the quality of the comparison in Table 12 or in the plot in Figure 1. at least for $k=3$. For $k=4$ and $t=10^{8}$, the terms $0 \leq r \leq 12$ all contribute to the first four digits of (52), and, again the quality of the fit to the actual moment would be noticeably worse if any of these terms were omitted. The numerics for $k=3$ and 4 alone provide substantial evidence favouring the full asymptotics of the moments, being sensitive to almost all the terms of the conjecture.

Our numerics for larger $k$ also support the conjectured moments, though are sensitive to a smaller set of terms of $P_{k}$ for the value $T$ examined. For example, when $k=7$, the $r=26$ term of $P_{k}$ contributes the most to (52). Furthermore, the first 2-3 digits of (52), for $k=7$ and $T=10^{8}$, arise from the terms with $19 \leq r \leq 33$, whereas the leading coefficient only contributes to around the 20th decimal place. This illustrates the importance, when testing the CFKRS prediction, of incorporating the lower terms of $P_{k}(x)$.

The paper of Hiary and Odlyzko $[\mathrm{HO}]$ contains additional numerics concerning the moments of zeta. Using values of $c_{r}(k)$ computed earlier in CFKRS, they examined the full asymptotics of the moments of $|\zeta(1 / 2+i t)|$, for $k \leq 6$, but for intervals $T \leq t \leq T+H$. Their values of $T$ are of size around $10^{7}, 10^{8}, 10^{15}, 10^{19}$, and $10^{22}$, while their values of $H$, are, for the most part, significantly smaller than $T$ or even small in comparison to $T^{1 / 2}$.

Their data shows, for intervals with $H$ quite small in comparison to $T^{1 / 2}$, a large amount of variation in the computed moments over various intervals of equal length $H$, whereas the predicted moments change very slowly as one varies $T$. Presumably one needs $H \gg T^{\beta}$ for some $\beta>1 / 2$ in order to get a good agreement between the computed and predicted moments. Indeed, their data set 's8', which is comparable to the interval of length approximately $10^{8}$ that we examined, shows excellent agreement between computed moments and predicted moments. Their dataset $z 16$, with $T \approx 10^{15}$ and $H \approx 10^{8}$ shows moderate agreement.

To calculate (51), we used the tanh-sinh quadrature scheme [Ba, BLJ] to accurately estimate each integral between consecutive non-trivial zeros of the zeta function on the critical line. All our computations of (51) were carried out using 64 bit machine doubles. To tabulate all the zeros up to, and slightly beyond, $T=10^{8}$, we used Rubinstein's C++ $L$-function package lcalc $[\mathrm{R}]$. It applies the Riemann Siegel formula to evaluate $\zeta(1 / 2+i t)$ and look for sign changes of the Hardy $Z$-function, Brent's method to compute the zeros of zeta $[\mathrm{Br}$, and a variant of Turing's test $\mathrm{E}]$ to confirm that all zeros up to given height have been found. 
Figures 1 and 2 depict the relative remainder term for 1000 values of $T$ between 0 and $10^{8}$, roughly spaced apart every $10^{5}$. More specifically, we let $T_{j}$ be the imaginary part of the first zero above $10^{5} j$, so that $T_{j} \approx 10^{5} j$, and plot the values of

$$
\frac{\operatorname{Data}_{k}\left(T_{j}\right)-\text { Conjecture }_{k}\left(T_{j}\right)}{\text { Conjecture }_{k}\left(T_{j}\right)},
$$

for $1 \leq j \leq 1000$, and $1 \leq k \leq 12$.

We also calculated the running average of the remainder term divided by the running average of the conjecture:

$$
\frac{\sum_{j=1}^{J}\left(\operatorname{Data}_{k}\left(T_{j}\right)-\text { Conjecture }_{k}\left(T_{j}\right)\right)}{\sum_{j=1}^{J} \text { Conjecture }_{k}\left(T_{j}\right)} .
$$

If we define

$$
\operatorname{SmoothData}_{k}(T)=\int_{T_{0}}^{\mathrm{T}}|\zeta(1 / 2+i t)|^{2 k}(1-t / T) d t
$$

and

$$
\text { SmoothConjecture }_{k}(T)=\int_{T_{0}}^{\mathrm{T}} P_{k}\left(\log \frac{t}{2 \pi}\right)(1-t / T) d t,
$$

then (54) gives a discrete approximation to the smoothed relative remainder:

$$
\frac{\operatorname{SmoothData}_{k}\left(T_{j}\right)-\text { SmoothConjecture }_{k}\left(T_{j}\right)}{\text { SmoothConjecture }_{k}\left(T_{j}\right)} .
$$

The reason for considering smoothed moments is that the noisy remainder terms of the sharply truncate moments, when averaged, tend to be smaller.

Note that the vertical axes in these figures change from plot to plot to allow us to meaningfully display the relative remainder terms, which as a whole get larger, as $k$ increases. We also set the zoom level to show the running averages in some detail. As a compromise, a few outliers are omitted from these plots for smaller $T$, roughly up to $10^{7}$, and $k \leq 4$.

Table 13 lists the standard deviations of 900 values of the remainder term and smoothed remainder term, for $1 \leq k \leq 13$. Specifically, we computed the standard deviation for the values of (53), $101 \leq j \leq 1000$, and of (57), for $101 \leq J \leq 1000$.

Table 12 lists the values of $\operatorname{Data}_{k}(T)$, and Conjecture $(T)$ for $k=1, \ldots, 13$ and $T=100000000.64$, the first zero of zeta above $10^{8}$. We also list the values of the averages over all 1000 values of $T_{j}$ :

$$
\frac{1}{1000} \sum_{j=1}^{1000} \operatorname{Data}_{k}\left(T_{j}\right)
$$

and

$$
\frac{1}{1000} \sum_{j=1}^{1000} \text { Conjecture }_{k}\left(T_{j}\right)
$$


Our data supports the CFKRS conjecture for the full asymptotics of the moments of zeta as described in equation (1), though for larger $k$, it is difficult to gauge the size of the remainder term.

For $k=1$ the data suggests an even stronger remainder term of $O\left(T^{1 / 4+\delta}\right)$, supported by the agreement of $\operatorname{Data}_{1}(T)$ with Conjecture $_{1}(T)$ to about $3 / 4$ of the decimal places left of the decimal point for the values of $T$ examined. The relative remainder term is of size around $10^{-6}$ when $T \approx 10^{8}$ and, typically, an order of magnitude smaller when averaged.

For $k=2$ the data agrees with the conjecture to about half the decimal places, with a relative remainder term of size around $10^{-6}$. For $k=3$, the agreement is to slightly less than half the decimal places.

For fixed $T$, as $k$ increases, the moments have the effect of amplifying the largest values of $|\zeta(1 / 2+i t)|$. This can be seen in our plots, for larger $k$, where the remainder terms are qualitatively the same, with large jumps at the same values of $T$ corresponding to relatively large values of zeta. It therefore becomes more difficult to ascertain, as $k$ grows, whether an upper bound for the remainder term of the form $O_{k}\left(T^{1 / 2+\delta}\right)$ holds. Nonetheless, the table and figures reveal an excellent fit for the CFKRS prediction with the moments which persists through to the 24th and 26th moments, where the relative agreement is to within around one to two decimal places.

In some sense, the fit between columns 2 and 3 of Table 12 is better than it ought to be for larger $k$, for example more than three decimal places for $k=13$, but only agreeing to around $90 \%$ for the running average of the remainder. A quick inspection of the figures reveals that the relative remainder, at $T=10^{8}$, happens to, fortuitously, best its neighbours, especially for larger $k$. Nonetheless, the overall agreement between the CFKRS prediction and our data across all values of $k$ and $T$, as depicted in the figures, lends strong support to their conjecture for the full asymptotics of the moments of the zeta function. 
TABle 2. Coefficients $c_{r}(k)$ for $k=4,5,6$. For $k=4,5$ we used $\delta=10^{-25}$, and $P=942939827,180343651$, respectively. For $k=6$ we combined two data sets using $\delta=10^{-25}, P=25501199$ for $0 \leq r \leq 29$, and $\delta=10^{-16}, P=608121859$ for $30 \leq r \leq 36$.

\begin{tabular}{|c|c|c|c|}
\hline$r$ & $c_{r}(4)$ & $c_{r}(5)$ & $c_{r}(6)$ \\
\hline 0 & $2.465018391934227354079894 \mathrm{e}-13$ & $1.416001020622731200955 \mathrm{e}-24$ & $5.12947340914919112 \mathrm{e}-40$ \\
\hline 1 & $5.450140573117186559363058 \mathrm{e}-11$ & $7.380412756494451305968 \mathrm{e}-22$ & $5.306732809926444246 \mathrm{e}-37$ \\
\hline 2 & $5.28772963479120311384897 \mathrm{e}-09$ & $1.7797796235196529053094 \mathrm{e}-19$ & $2.6079207711483512396 \mathrm{e}-34$ \\
\hline 3 & $2.96411431799939794596918 \mathrm{e}-07$ & $2.635886609660724758286 \mathrm{e}-17$ & $8.1016132157790177281 \mathrm{e}-32$ \\
\hline 4 & $1.064595006812847051321182 \mathrm{e}-05$ & $2.6840545349997485760134 \mathrm{e}-15$ & $1.7861297380093099773 \mathrm{e}-29$ \\
\hline 5 & $2.5702983342426340235494 \mathrm{e}-04$ & $1.993641309249897180312 \mathrm{e}-13$ & $2.9743167108636063482 \mathrm{e}-27$ \\
\hline 6 & $4.2639216163116947218762 \mathrm{e}-03$ & $1.1184855124933629437778 \mathrm{e}-11$ & $3.8877082911558678876 \mathrm{e}-25$ \\
\hline 7 & $4.89414245142160102712761 \mathrm{e}-02$ & $4.842797553044804165519 \mathrm{e}-10$ & $4.09224261406862935514 \mathrm{e}-23$ \\
\hline 8 & $3.878526654019553499833 \mathrm{e}-01$ & $1.639801308496156099797 \mathrm{e}-08$ & $3.5314663856570325725 \mathrm{e}-21$ \\
\hline 9 & $2.10913382864873355204 \mathrm{e}+00$ & $4.374935105492246330412 \mathrm{e}-07$ & $2.530637690060973478289 \mathrm{e}-19$ \\
\hline 10 & $7.8325356118822623579303 \mathrm{e}+00$ & $9.22633350296530326337 \mathrm{e}-06$ & $1.5198191029685924995 \mathrm{e}-17$ \\
\hline 11 & $1.982806812499890923 \mathrm{e}+01$ & $1.537677778207107946991 \mathrm{e}-04$ & $7.70015137609237458270 \mathrm{e}-16$ \\
\hline 12 & $3.388893203738368856 \mathrm{e}+01$ & $2.01902775807813195907 \mathrm{e}-03$ & $3.3061210414107436046 \mathrm{e}-14$ \\
\hline 13 & $3.82033062189019517 \mathrm{e}+01$ & $2.07727067284846475474 \mathrm{e}-02$ & $1.2064041518984715612 \mathrm{e}-12$ \\
\hline 14 & $2.560441501227035 \mathrm{e}+01$ & $1.6625058643910393652 \mathrm{e}-01$ & $3.7467192541626917996 \mathrm{e}-11$ \\
\hline 15 & $1.06189693794016 \mathrm{e}+01$ & $1.026466777849473756 \mathrm{e}+00$ & $9.9056942856889097902 \mathrm{e}-10$ \\
\hline 16 & $7.089464552244 \mathrm{e}-01$ & $4.848589278343642478 \mathrm{e}+00$ & $2.2273885767179683823 \mathrm{e}-08$ \\
\hline 17 & & $1.73908760901310234 \mathrm{e}+01$ & $4.251372866816786076 \mathrm{e}-07$ \\
\hline 18 & & $4.7040877087561734 \mathrm{e}+01$ & $6.8674335769870947550 \mathrm{e}-06$ \\
\hline 19 & & $9.511661794587886 \mathrm{e}+01$ & $9.351583018775044262 \mathrm{e}-05$ \\
\hline 20 & & $1.41444460064317 \mathrm{e}+02$ & $1.068316421173022528 \mathrm{e}-03$ \\
\hline 21 & & $1.4935694999630 \mathrm{e}+02$ & $1.01807023862361485 \mathrm{e}-02$ \\
\hline 22 & & $1.0588728028422 \mathrm{e}+02$ & $8.04186793058379244 \mathrm{e}-02$ \\
\hline 23 & & $4.41362307288 \mathrm{e}+01$ & $5.2296141941724947 \mathrm{e}-01$ \\
\hline 24 & & $2.010650046 \mathrm{e}+01$ & $2.7802017665195719 \mathrm{e}+00$ \\
\hline 25 & & $-1.2701703 \mathrm{e}+00$ & $1.200111408801811 \mathrm{e}+01$ \\
\hline 26 & & & $4.179670936891264 \mathrm{e}+01$ \\
\hline 27 & & & $1.16723095829484 \mathrm{e}+02$ \\
\hline 28 & & & $2.5939897299715 \mathrm{e}+02$ \\
\hline 29 & & & $4.524908135220 \mathrm{e}+02$ \\
\hline 30 & & & $6.0117334836510 \mathrm{e}+02$ \\
\hline 31 & & & $5.7354384553122 \mathrm{e}+02$ \\
\hline 32 & & & $3.75018676133 \mathrm{e}+02$ \\
\hline 33 & & & $2.46890415605 \mathrm{e}+02$ \\
\hline 34 & & & $2.454954369 \mathrm{e}+02$ \\
\hline 35 & & & $1.603303769 \mathrm{e}+02$ \\
\hline 36 & & & $-3.78219665 \mathrm{e}+01$ \\
\hline
\end{tabular}


TABle 3. Coefficients for $k=7$ truncating at $P=11015647$, and using $\delta=10^{-16}$.

\begin{tabular}{|c|c||c|c||c|c|}
\hline$r$ & $c_{r}(7)$ & $r$ & $c_{r}(7)$ & $r$ & $c_{r}(7)$ \\
\hline 0 & $6.5822847876005500 \mathrm{e}-60$ & 1 & $1.2041430555451870 \mathrm{e}-56$ & 2 & $1.0621355717492720 \mathrm{e}-53$ \\
3 & $6.0172653760159300 \mathrm{e}-51$ & 4 & $2.4606287673240130 \mathrm{e}-48$ & 5 & $7.7390121665211530 \mathrm{e}-46$ \\
6 & $1.9478649494952360 \mathrm{e}-43$ & 7 & $4.0307684926363700 \mathrm{e}-41$ & 8 & $6.9917763337237880 \mathrm{e}-39$ \\
9 & $1.0314019779812270 \mathrm{e}-36$ & 10 & $1.3082869144993580 \mathrm{e}-34$ & 11 & $1.4392681201435320 \mathrm{e}-32$ \\
12 & $1.3825312154986080 \mathrm{e}-30$ & 13 & $1.1657759371318020 \mathrm{e}-28$ & 14 & $8.6652476933527220 \mathrm{e}-27$ \\
15 & $5.6962227424753780 \mathrm{e}-25$ & 16 & $3.3197648540507990 \mathrm{e}-23$ & 17 & $1.7183970393294220 \mathrm{e}-21$ \\
18 & $7.9096788893235440 \mathrm{e}-20$ & 19 & $3.2396929335740840 \mathrm{e}-18$ & 20 & $1.1809579273268370 \mathrm{e}-16$ \\
21 & $3.8302270051510130 \mathrm{e}-15$ & 22 & $1.1044706290361260 \mathrm{e}-13$ & 23 & $2.8282258231583490 \mathrm{e}-12$ \\
24 & $6.4210662257609940 \mathrm{e}-11$ & 25 & $1.2898755567219640 \mathrm{e}-09$ & 26 & $2.2869667400876520 \mathrm{e}-08$ \\
27 & $3.5683995004969530 \mathrm{e}-07$ & 28 & $4.8834071041615500 \mathrm{e}-06$ & 29 & $5.8391045220798220 \mathrm{e}-05$ \\
30 & $6.0742037327532430 \mathrm{e}-04$ & 31 & $5.4716438254364890 \mathrm{e}-03$ & 32 & $4.2465903403750590 \mathrm{e}-02$ \\
33 & $2.824549346789606 \mathrm{e}-01$ & 34 & $1.601333066518585 \mathrm{e}+00$ & 35 & $7.696699614092694 \mathrm{e}+00$ \\
36 & $3.12035202072577 \mathrm{e}+01$ & 37 & $1.06197143546798 \mathrm{e}+02$ & 38 & $3.019136554174 \mathrm{e}+02$ \\
39 & $7.117410357280 \mathrm{e}+02$ & 40 & $1.37009445510 \mathrm{e}+03$ & 41 & $2.0827987442 \mathrm{e}+03$ \\
42 & $2.357363536 \mathrm{e}+03$ & 43 & $1.93463843 \mathrm{e}+03$ & 44 & $1.75714310 \mathrm{e}+03$ \\
45 & $2.853378 \mathrm{e}+03$ & 46 & $3.100593 \mathrm{e}+03$ & 47 & $3.3940 \mathrm{e}+02$ \\
48 & $-1.20854 \mathrm{e}+03$ & 49 & $-5.0194 \mathrm{e}+02$ & & \\
\hline
\end{tabular}

TABle 4. Coefficients for $k=8$ truncating at $P=1212569$, and using $\delta=10^{-16}$.

\begin{tabular}{|c|c||c|c||c|c|}
\hline$r$ & $c_{r}(8)$ & $r$ & $c_{r}(8)$ & $r$ & $c_{r}(8)$ \\
\hline 0 & $1.870442160117 \mathrm{e}-84$ & 1 & $5.570219365179 \mathrm{e}-81$ & 2 & $8.0727983790767 \mathrm{e}-78$ \\
3 & $7.5876025208718 \mathrm{e}-75$ & 4 & $5.2002464291967 \mathrm{e}-72$ & 5 & $2.7705098412043 \mathrm{e}-69$ \\
6 & $1.1944832708049 \mathrm{e}-66$ & 7 & $4.28399526987474 \mathrm{e}-64$ & 8 & $1.30388783552972 \mathrm{e}-61$ \\
9 & $3.41901547588078 \mathrm{e}-59$ & 10 & $7.814883564309408 \mathrm{e}-57$ & 11 & $1.5716390984187150 \mathrm{e}-54$ \\
12 & $2.8019878105779590 \mathrm{e}-52$ & 13 & $4.455999566980015 \mathrm{e}-50$ & 14 & $6.353422665784412 \mathrm{e}-48$ \\
15 & $8.1564318452061200 \mathrm{e}-46$ & 16 & $9.461598292152723 \mathrm{e}-44$ & 17 & $9.946939863498895 \mathrm{e}-42$ \\
18 & $9.5005755608004230 \mathrm{e}-40$ & 19 & $8.2610748506972050 \mathrm{e}-38$ & 20 & $6.5505802739681480 \mathrm{e}-36$ \\
21 & $4.7431624904297110 \mathrm{e}-34$ & 22 & $3.1395134797176380 \mathrm{e}-32$ & 23 & $1.9011136618426250 \mathrm{e}-30$ \\
24 & $1.0537646192031000 \mathrm{e}-28$ & 25 & $5.3481979466238930 \mathrm{e}-27$ & 26 & $2.4856705239072160 \mathrm{e}-25$ \\
27 & $1.0578033941072590 \mathrm{e}-23$ & 28 & $4.1205620801239040 \mathrm{e}-22$ & 29 & $1.4685071632966620 \mathrm{e}-20$ \\
30 & $4.7847077672871950 \mathrm{e}-19$ & 31 & $1.4239651481852300 \mathrm{e}-17$ & 32 & $3.8665596212165030 \mathrm{e}-16$ \\
33 & $9.566613928613862 \mathrm{e}-15$ & 34 & $2.1534462266724710 \mathrm{e}-13$ & 35 & $4.4024065808181710 \mathrm{e}-12$ \\
36 & $8.1576484690923080 \mathrm{e}-11$ & 37 & $1.367077736688555 \mathrm{e}-09$ & 38 & $2.0668154599686450 \mathrm{e}-08$ \\
39 & $2.811291057443101 \mathrm{e}-07$ & 40 & $3.430097716487554 \mathrm{e}-06$ & 41 & $3.741873571285350 \mathrm{e}-05$ \\
42 & $3.63684791980377 \mathrm{e}-04$ & 43 & $3.137462556407600 \mathrm{e}-03$ & 44 & $2.39287182393225 \mathrm{e}-02$ \\
45 & $1.60675070999268 \mathrm{e}-01$ & 46 & $9.4588131743684 \mathrm{e}-01$ & 47 & $4.8616355021107 \mathrm{e}+00$ \\
48 & $2.173103986560 \mathrm{e}+01$ & 49 & $8.417133459453 \mathrm{e}+01$ & 50 & $2.81517268211 \mathrm{e}+02$ \\
51 & $8.0929177383 \mathrm{e}+02$ & 52 & $1.9821841216 \mathrm{e}+03$ & 53 & $4.05873574 \mathrm{e}+03$ \\
54 & $6.69566487 \mathrm{e}+03$ & 55 & $8.4203977 \mathrm{e}+03$ & 56 & $8.096360 \mathrm{e}+03$ \\
57 & $9.4961243 \mathrm{e}+03$ & 58 & $1.99106 \mathrm{e}+04$ & 59 & $3.09087 \mathrm{e}+04$ \\
60 & $1.3133 \mathrm{e}+04$ & 61 & $-2.964 \mathrm{e}+04$ & 62 & $-4.0582 \mathrm{e}+04$ \\
63 & $-8.56 \mathrm{e}+03$ & 64 & $4.56 \mathrm{e}+03$ & & \\
\hline & & & & \\
& & &
\end{tabular}


TABle 5. Coefficients for $k=9$ truncating at $P=170741$, and using $\delta=10^{-16}$.

\begin{tabular}{|c|c||c|c||c|c|}
\hline$r$ & $c_{r}(9)$ & $r$ & $c_{r}(9)$ & $r$ & $c_{r}(9)$ \\
\hline 0 & $7.920155238 \mathrm{e}-114$ & 1 & $3.608743873 \mathrm{e}-110$ & 2 & $8.051296272 \mathrm{e}-107$ \\
3 & $1.172362406 \mathrm{e}-103$ & 4 & $1.2530058769 \mathrm{e}-100$ & 5 & $1.0481427376 \mathrm{e}-97$ \\
6 & $7.1456310032 \mathrm{e}-95$ & 7 & $4.0821744596 \mathrm{e}-92$ & 8 & $1.9941925290 \mathrm{e}-89$ \\
9 & $8.4594205088 \mathrm{e}-87$ & 10 & $3.1538144173 \mathrm{e}-84$ & 11 & $1.0433789942 \mathrm{e}-81$ \\
12 & $3.08731306439 \mathrm{e}-79$ & 13 & $8.22414128961 \mathrm{e}-77$ & 14 & $1.98314875716 \mathrm{e}-74$ \\
15 & $4.34906593630 \mathrm{e}-72$ & 16 & $8.70843432097 \mathrm{e}-70$ & 17 & $1.597618788502 \mathrm{e}-67$ \\
18 & $2.693264006511 \mathrm{e}-65$ & 19 & $4.1828312947266 \mathrm{e}-63$ & 20 & $5.9981084135426 \mathrm{e}-61$ \\
21 & $7.9570365567690 \mathrm{e}-59$ & 22 & $9.7816383607331 \mathrm{e}-57$ & 23 & $1.1159063652824 \mathrm{e}-54$ \\
24 & $1.1828936996292 \mathrm{e}-52$ & 25 & $1.16636205776320 \mathrm{e}-50$ & 26 & $1.0707494871812 \mathrm{e}-48$ \\
27 & $9.1588630961799 \mathrm{e}-47$ & 28 & $7.30410594473147 \mathrm{e}-45$ & 29 & $5.43352058419794 \mathrm{e}-43$ \\
30 & $3.77180308962311 \mathrm{e}-41$ & 31 & $2.44391067332666 \mathrm{e}-39$ & 32 & $1.47828492081742 \mathrm{e}-37$ \\
33 & $8.34814115852835 \mathrm{e}-36$ & 34 & $4.401070135763961 \mathrm{e}-34$ & 35 & $2.16570032669324 \mathrm{e}-32$ \\
36 & $9.94493396339446 \mathrm{e}-31$ & 37 & $4.26008480938235 \mathrm{e}-29$ & 38 & $1.7015865979528490 \mathrm{e}-27$ \\
39 & $6.33392559975936 \mathrm{e}-26$ & 40 & $2.19581041600330 \mathrm{e}-24$ & 41 & $7.08426675189073 \mathrm{e}-23$ \\
42 & $2.1252127802020740 \mathrm{e}-21$ & 43 & $5.92241988702831 \mathrm{e}-20$ & 44 & $1.53150283306451 \mathrm{e}-18$ \\
45 & $3.670620764266784 \mathrm{e}-17$ & 46 & $8.14314143690125 \mathrm{e}-16$ & 47 & $1.66973151449710 \mathrm{e}-14$ \\
48 & $3.15948278435009 \mathrm{e}-13$ & 49 & $5.507449862131347 \mathrm{e}-12$ & 50 & $8.82746086945955 \mathrm{e}-11$ \\
51 & $1.29834184462004 \mathrm{e}-09$ & 52 & $1.74846308044295 \mathrm{e}-08$ & 53 & $2.1508513613974 \mathrm{e}-07$ \\
54 & $2.4107241046116 \mathrm{e}-06$ & 55 & $2.4552071751795 \mathrm{e}-05$ & 56 & $2.265572158192 \mathrm{e}-04$ \\
57 & $1.888394621727 \mathrm{e}-03$ & 58 & $1.4172609520338 \mathrm{e}-02$ & 59 & $9.546112504952 \mathrm{e}-02$ \\
60 & $5.751640191248 \mathrm{e}-01$ & 61 & $3.08994036050 \mathrm{e}+00$ & 62 & $1.47569216973 \mathrm{e}+01$ \\
63 & $6.2480977623 \mathrm{e}+01$ & 64 & $2.3393469844 \mathrm{e}+02$ & 65 & $7.721276404 \mathrm{e}+02$ \\
66 & $2.23430645 \mathrm{e}+03$ & 67 & $5.60365351 \mathrm{e}+03$ & 68 & $1.1907448 \mathrm{e}+04$ \\
69 & $2.062257 \mathrm{e}+04$ & 70 & $2.776900 \mathrm{e}+04$ & 71 & $3.06185 \mathrm{e}+04$ \\
72 & $4.7187 \mathrm{e}+04$ & 73 & $1.2202 \mathrm{e}+05$ & 74 & $2.314 \mathrm{e}+05$ \\
75 & $1.255 \mathrm{e}+05$ & 76 & $-4.658 \mathrm{e}+05$ & 77 & $-1.07 \mathrm{e}+06$ \\
78 & $-5.794 \mathrm{e}+05$ & 79 & $6.75 \mathrm{e}+05$ & 80 & $8.27 \mathrm{e}+05$ \\
81 & $1.3 \mathrm{e}+05$ & & & & \\
\hline
\end{tabular}


TABle 6 . Coefficients for $k=10$ truncating at $P=675929$, and using $\delta=10^{-16}$.

\begin{tabular}{|c|c|c|c|c|c|}
\hline$r$ & $c_{r}(10)$ & $r$ & $c_{r}(10)$ & $r$ & $c_{r}(10)$ \\
\hline 0 & $3.54888492477 \mathrm{e}-148$ & 1 & $2.35769133101 \mathrm{e}-144$ & 2 & $7.70233663026 \mathrm{e}-141$ \\
\hline 3 & $1.64948634407 \mathrm{e}-137$ & 4 & $2.60451944693 \mathrm{e}-134$ & 5 & $3.23366677841 \mathrm{e}-131$ \\
\hline 6 & $3.28765141574 \mathrm{e}-128$ & 7 & $2.81472946999 \mathrm{e}-125$ & 8 & $2.07111222708 \mathrm{e}-122$ \\
\hline 9 & $1.330223430450 \mathrm{e}-119$ & 10 & $7.54902089850 \mathrm{e}-117$ & 11 & $3.822610704580 \mathrm{e}-114$ \\
\hline 12 & $1.741117024160 \mathrm{e}-111$ & 13 & 7.181397221310e-109 & 14 & $2.697527199940 \mathrm{e}-106$ \\
\hline 15 & $9.272621188300 \mathrm{e}-104$ & 16 & $2.929091296840 \mathrm{e}-101$ & 17 & $8.533614631040 \mathrm{e}-99$ \\
\hline 18 & $2.300282495690 \mathrm{e}-96$ & 19 & $5.752933603890 \mathrm{e}-94$ & 20 & $1.338222245340 \mathrm{e}-91$ \\
\hline 21 & $2.901664262540 \mathrm{e}-89$ & 22 & $5.876116434230 \mathrm{e}-87$ & 23 & $1.113295629950 \mathrm{e}-84$ \\
\hline 24 & $1.976420578280 \mathrm{e}-82$ & 25 & $3.292284406610 \mathrm{e}-80$ & 26 & $5.152298303310 \mathrm{e}-78$ \\
\hline 27 & $7.583498206660 \mathrm{e}-76$ & 28 & $1.050825438590 \mathrm{e}-73$ & 29 & $1.372032297570 \mathrm{e}-71$ \\
\hline 30 & $1.689305278750 \mathrm{e}-69$ & 31 & $1.962725749490 \mathrm{e}-67$ & 32 & $2.153176878340 \mathrm{e}-65$ \\
\hline 33 & $2.231491144780 \mathrm{e}-63$ & 34 & $2.185751487190 \mathrm{e}-61$ & 35 & $2.024236345360 \mathrm{e}-59$ \\
\hline 36 & $1.773015880790 \mathrm{e}-57$ & 37 & $1.469142759970 \mathrm{e}-55$ & 38 & $1.151856372240 \mathrm{e}-53$ \\
\hline 39 & $8.546210010780 \mathrm{e}-52$ & 40 & $6.000996648940 \mathrm{e}-50$ & 41 & $3.988024973250 \mathrm{e}-48$ \\
\hline 42 & $2.508210346100 \mathrm{e}-46$ & 43 & $1.492814370840 \mathrm{e}-44$ & 44 & $8.406690331770 \mathrm{e}-43$ \\
\hline 45 & $4.478585581910 \mathrm{e}-41$ & 46 & $2.256576452310 \mathrm{e}-39$ & 47 & $1.075045360230 \mathrm{e}-37$ \\
\hline 48 & $4.840860160570 \mathrm{e}-36$ & 49 & $2.059518250610 \mathrm{e}-34$ & 50 & $8.274865115540 \mathrm{e}-33$ \\
\hline 51 & $3.138261284280 \mathrm{e}-31$ & 52 & $1.122809702480 \mathrm{e}-29$ & 53 & $3.787412159890 \mathrm{e}-28$ \\
\hline 54 & $1.203656074660 \mathrm{e}-26$ & 55 & $3.601325369860 \mathrm{e}-25$ & 56 & $1.013609245800 \mathrm{e}-23$ \\
\hline 57 & $2.681299896780 \mathrm{e}-22$ & 58 & $6.659997348320 \mathrm{e}-21$ & 59 & $1.551711921570 \mathrm{e}-19$ \\
\hline 60 & $3.387479938260 \mathrm{e}-18$ & 61 & $6.920780548090 \mathrm{e}-17$ & 62 & $1.321576287680 \mathrm{e}-15$ \\
\hline 63 & $2.355560477620 \mathrm{e}-14$ & 64 & $3.913136454580 \mathrm{e}-13$ & 65 & $6.049282423090 \mathrm{e}-12$ \\
\hline 66 & $8.687675538270 \mathrm{e}-11$ & 67 & $1.157038978680 \mathrm{e}-09$ & 68 & $1.426296610210 \mathrm{e}-08$ \\
\hline 69 & $1.624086810140 \mathrm{e}-07$ & 70 & $1.704560517010 \mathrm{e}-06$ & 71 & $1.645250259970 \mathrm{e}-05$ \\
\hline 72 & $1.456896715700 \mathrm{e}-04$ & 73 & $1.180639233900 \mathrm{e}-03$ & 74 & $8.733236736800 \mathrm{e}-03$ \\
\hline 75 & $5.881138307580 \mathrm{e}-02$ & 76 & $3.596179964360 \mathrm{e}-01$ & 77 & $1.991704581680 \mathrm{e}+00$ \\
\hline 78 & $9.96798553888 \mathrm{e}+00$ & 79 & $4.49881033284 \mathrm{e}+01$ & 80 & $1.8275974779 \mathrm{e}+02$ \\
\hline 81 & $6.6682890321 \mathrm{e}+02$ & 82 & $2.177262632 \mathrm{e}+03$ & 83 & $6.31448075 \mathrm{e}+03$ \\
\hline 84 & $1.60290666 \mathrm{e}+04$ & 85 & $3.47003569 \mathrm{e}+04$ & 86 & $6.163495 \mathrm{e}+04$ \\
\hline 87 & $8.726963 \mathrm{e}+04$ & 88 & $1.147239 \mathrm{e}+05$ & 89 & $2.48873 \mathrm{e}+05$ \\
\hline 90 & $7.40512 \mathrm{e}+05$ & 91 & $1.4296 \mathrm{e}+06$ & 92 & $2.56 \mathrm{e}+05$ \\
\hline 93 & $-6.2748 \mathrm{e}+06$ & 94 & $-1.489 \mathrm{e}+07$ & 95 & $-7.97 \mathrm{e}+06$ \\
\hline 96 & $2.260 \mathrm{e}+07$ & 97 & $4.02 \mathrm{e}+07$ & 98 & $1.43 \mathrm{e}+07$ \\
\hline 99 & $-1.08 \mathrm{e}+07$ & 100 & $-5.22 \mathrm{e}+06$ & & \\
\hline
\end{tabular}


TABLE 7. Coefficients for $k=11$ truncating at $P=85889$, and using $\delta=10^{-12}$.

\begin{tabular}{|c|c|c|c|c|c|c|c|}
\hline$r$ & $c_{r}(11)$ & $r$ & $c_{r}(11)$ & $r$ & $c_{r}(11)$ & $r$ & $c_{r}(11)$ \\
\hline 0 & $1.2451314 \mathrm{e}-187$ & 1 & $1.16057289 \mathrm{e}-183$ & 2 & $5.33593693 \mathrm{e}-180$ & 3 & $1.61328064 \mathrm{e}-176$ \\
\hline 4 & $3.60796892 \mathrm{e}-173$ & 5 & $6.36556626 \mathrm{e}-170$ & 6 & $9.22782529 \mathrm{e}-167$ & 7 & $1.13037084 \mathrm{e}-163$ \\
\hline 8 & $1.19424156 \mathrm{e}-160$ & 9 & $1.10531689 \mathrm{e}-157$ & 10 & $9.07262368 \mathrm{e}-155$ & 11 & $6.67001789 \mathrm{e}-152$ \\
\hline 12 & $4.42795938 \mathrm{e}-149$ & 13 & $2.67250329 \mathrm{e}-146$ & 14 & $1.47494511 \mathrm{e}-143$ & 15 & $7.48038633 \mathrm{e}-141$ \\
\hline 16 & $3.50123968 \mathrm{e}-138$ & 17 & 1.51807108 & 18 & $6.117325360 \mathrm{e}-133$ & 19 & 2.297695573 \\
\hline 20 & $8.065058896 \mathrm{e}-128$ & 21 & 2.65161261 & 22 & $8.182770981 \mathrm{e}-123$ & 23 & 2.37459325 \\
\hline 24 & 6.490958842 & 25 & 1.673872653 & 26 & 4.077854544 & 27 & 9.39688582 \\
\hline 28 & $2.050582239 \mathrm{e}-108$ & 29 & 4.241948499 & 30 & 8.3264998 & 31 & 1.552193 \\
\hline 32 & $2.7501740419 \mathrm{e}-99$ & 33 & $4.6346698213 \mathrm{e}-97$ & 34 & $7.4337414866 \mathrm{e}-95$ & 35 & 1.13549625 \\
\hline 36 & $1.65268057424 \mathrm{e}-90$ & 37 & $2.29313645275 \mathrm{e}-88$ & 38 & 3.03459182 & 39 & $3.831526640190 \mathrm{e}-84$ \\
\hline 40 & $4.617401084980 \mathrm{e}-82$ & 41 & $5.312669020390 \mathrm{e}-80$ & 42 & $5.837617513170 \mathrm{e}-78$ & 43 & $6.127289274640 \mathrm{e}-76$ \\
\hline 44 & $6.144657440350 \mathrm{e}-74$ & 45 & $5.888372616130 \mathrm{e}-72$ & 46 & $5.392853053390 \mathrm{e}-70$ & 47 & $4.720750019910 \mathrm{e}-68$ \\
\hline 48 & $3.950047958650 \mathrm{e}-66$ & 49 & $3.1594336:$ & 50 & 2.415656 & 51 & $1.765512476040 \mathrm{e}-60$ \\
\hline 52 & $1.233372385020 \mathrm{e}-58$ & 53 & $8.235138259720 \mathrm{e}-57$ & 54 & $5.254770626530 \mathrm{e}-55$ & 55 & $3.203931860340 \mathrm{e}-53$ \\
\hline 56 & $1.866325220220 \mathrm{e}-51$ & 57 & $1.038439545240 \mathrm{e}-49$ & 58 & $5.517819788010 \mathrm{e}-48$ & 59 & $2.799212525590 \mathrm{e}-46$ \\
\hline 60 & $1.355385595070 \mathrm{e}-44$ & 61 & $6.261980454760 \mathrm{e}-43$ & 62 & $2.759510005390 \mathrm{e}-41$ & 63 & $1.159469298800 \mathrm{e}-39$ \\
\hline 64 & $4.643172007710 \mathrm{e}-38$ & 65 & $1.771352314140 \mathrm{e}-36$ & 66 & $6.434590479390 \mathrm{e}-35$ & 67 & $2.224528434850 \mathrm{e}-33$ \\
\hline 68 & $7.315010837230 \mathrm{e}-32$ & 69 & $2.286618699140 \mathrm{e}-30$ & 70 & $6.790446084110 \mathrm{e}-29$ & 71 & $1.914406176510 \mathrm{e}-27$ \\
\hline 72 & $5.120202491620 \mathrm{e}-26$ & 73 & $1.298144910520 \mathrm{e}-24$ & 74 & $3.117357525500 \mathrm{e}-23$ & 75 & $7.084341566220 \mathrm{e}-22$ \\
\hline 76 & $1.522160095850 \mathrm{e}-20$ & 77 & $3.089177852240 \mathrm{e}-19$ & 78 & $5.915547597220 \mathrm{e}-18$ & 79 & $1.067669213960 \mathrm{e}-16$ \\
\hline 80 & $1.814092977890 \mathrm{e}-15$ & 81 & $2.898172629520 \mathrm{e}-14$ & 82 & $4.347699833250 \mathrm{e}-13$ & 83 & $6.115899813510 \mathrm{e}-12$ \\
\hline 84 & $8.055381828660 \mathrm{e}-11$ & 85 & $9.918802503370 \mathrm{e}-10$ & 86 & $1.139891722860 \mathrm{e}-08$ & 87 & $1.220516286130 \mathrm{e}-07$ \\
\hline 88 & $1.215356440380 \mathrm{e}-06$ & 89 & $1.123334898600 \mathrm{e}-05$ & 90 & $9.618099818200 \mathrm{e}-05$ & 91 & $7.612754647750 \mathrm{e}-04$ \\
\hline 92 & $5.558326140280 \mathrm{e}-03$ & 93 & $3.73566507420 \mathrm{e}-02$ & 94 & $2.3062374960 \mathrm{e}-01$ & 95 & $1.3052451738 \mathrm{e}+00$ \\
\hline 96 & $6.7601261642 \mathrm{e}+00$ & 97 & $3.199030312 \mathrm{e}+01$ & 98 & $1.381287107 \mathrm{e}+02$ & 99 & $5.43353076 \mathrm{e}+02$ \\
\hline 100 & $1.94226636 \mathrm{e}+03$ & 101 & $6.2767681 \mathrm{e}+03$ & 102 & $1.8153278 \mathrm{e}+04$ & 103 & $4.6140024 \mathrm{e}+04$ \\
\hline 104 & $1.001842 \mathrm{e}+05$ & 105 & $1.79901 \mathrm{e}+05$ & 106 & $2.732785 \mathrm{e}+05$ & 107 & $4.8073 \mathrm{e}+05$ \\
\hline 108 & $1.4164 \mathrm{e}+06$ & 109 & $4.18178 \mathrm{e}+06$ & 110 & $6.523 \mathrm{e}+06$ & 111 & $-7.31 \mathrm{e}+06$ \\
\hline 112 & $-6.295 \mathrm{e}+07$ & 113 & $-1.26 \mathrm{e}+08$ & 114 & $-1.2 \mathrm{e}+07$ & 115 & $4.21 \mathrm{e}+08$ \\
\hline 116 & $7.32 \mathrm{e}+08$ & 117 & $1.7 \mathrm{e}+08$ & 118 & $-8.1 e+08$ & 119 & $-8.2 \mathrm{e}+08$ \\
\hline 120 & $-1.1 \mathrm{e}+08$ & 121 & $9.9 \mathrm{e}+07$ & & & & \\
\hline
\end{tabular}


TABle 8. Coefficients for $k=12$ truncating at $P=12979$, and using $\delta=10^{-12}$.

\begin{tabular}{|c|c|c|c|c|c|c|c|}
\hline$r$ & $c_{r}(12)$ & $r$ & $c_{r}(12)$ & $r$ & $c_{r}(12)$ & $r$ & $c_{r}(12)$ \\
\hline 0 & $2.61438 \mathrm{e}-232$ & 1 & $3.31314 \mathrm{e}-228$ & 2 & $2.07583 \mathrm{e}-224$ & 3 & $8.57284 \mathrm{e}-221$ \\
\hline 4 & $2.62512 \mathrm{e}-217$ & 5 & $6.35698 \mathrm{e}-214$ & 6 & $1.26799 \mathrm{e}-210$ & 7 & $2.14259 \mathrm{e}-207$ \\
\hline 8 & $3.13061 \mathrm{e}-204$ & 9 & $4.01773 \mathrm{e}-201$ & 10 & $4.58505 \mathrm{e}-198$ & 11 & $4.69936 \mathrm{e}-195$ \\
\hline 12 & $4.36135 \mathrm{e}-192$ & 13 & $3.69038 \mathrm{e}-189$ & 14 & $2.86364 \mathrm{e}-186$ & 15 & $2.04802 \mathrm{e}-183$ \\
\hline 16 & $1.35583 \mathrm{e}-180$ & 17 & $8.34021 \mathrm{e}-178$ & 18 & $4.78307 \mathrm{e}-175$ & 19 & $2.56498 \mathrm{e}-172$ \\
\hline 20 & $1.28962 \mathrm{e}-169$ & 21 & $6.09352 \mathrm{e}-167$ & 22 & $2.71168 \mathrm{e}-164$ & 23 & $1.13871 \mathrm{e}-161$ \\
\hline 24 & $4.52018 \mathrm{e}-159$ & 25 & $1.698887 \mathrm{e}-156$ & 26 & $6.054452 \mathrm{e}-154$ & 27 & $2.048658 \mathrm{e}-151$ \\
\hline 28 & $6.589967 \mathrm{e}-149$ & 29 & $2.017472 \mathrm{e}-146$ & 30 & $5.884306 \mathrm{e}-144$ & 31 & $1.636682 \mathrm{e}-141$ \\
\hline 32 & 4.345107e-139 & 33 & $1.101947 \mathrm{e}-136$ & 34 & $2.671618 \mathrm{e}-134$ & 35 & $6.196518 \mathrm{e}-132$ \\
\hline 36 & $1.375825 \mathrm{e}-129$ & 37 & $2.926060 \mathrm{e}-127$ & 38 & $5.964172 \mathrm{e}-125$ & 39 & $1.165705 \mathrm{e}-122$ \\
\hline 40 & $2.185779 \mathrm{e}-120$ & 41 & $3.9336446 \mathrm{e}-118$ & 42 & $6.7972368 \mathrm{e}-116$ & 43 & $1.1281875 \mathrm{e}-113$ \\
\hline 44 & $1.7992516 \mathrm{e}-111$ & 45 & $2.7580481 \mathrm{e}-109$ & 46 & $4.0647787 \mathrm{e}-107$ & 47 & $5.7611834 \mathrm{e}-105$ \\
\hline 48 & $7.8547080 \mathrm{e}-103$ & 49 & $1.0303518 \mathrm{e}-100$ & 50 & $1.3006541 \mathrm{e}-98$ & 51 & $1.58027693 \mathrm{e}-96$ \\
\hline 52 & $1.84826712 \mathrm{e}-94$ & 53 & $2.08119455 \mathrm{e}-92$ & 54 & $2.25644465 \mathrm{e}-90$ & 55 & $2.35580895 \mathrm{e}-88$ \\
\hline 56 & $2.36859061 \mathrm{e}-86$ & 57 & $2.293494547 \mathrm{e}-84$ & 58 & $2.138841512 \mathrm{e}-82$ & 59 & $1.921057763 \mathrm{e}-80$ \\
\hline 60 & $1.6618175746 \mathrm{e}-78$ & 61 & $1.3845226981 \mathrm{e}-76$ & 62 & $1.110902429 \mathrm{e}-74$ & 63 & $8.583981848 \mathrm{e}-73$ \\
\hline 64 & $6.3871821912 \mathrm{e}-71$ & 65 & $4.5761550941 \mathrm{e}-69$ & 66 & $3.1565961813 \mathrm{e}-67$ & 67 & $2.09610369144 \mathrm{e}-65$ \\
\hline 68 & $1.33974800679 \mathrm{e}-63$ & 69 & $8.2410737927 \mathrm{e}-62$ & 70 & $4.8777660410 \mathrm{e}-60$ & 71 & $2.7774938619 \mathrm{e}-58$ \\
\hline 72 & $1.5212162503 \mathrm{e}-56$ & 73 & $8.01193333444 \mathrm{e}-55$ & 74 & $4.05683148804 \mathrm{e}-53$ & 75 & $1.97436017587 \mathrm{e}-51$ \\
\hline 76 & $9.23280422076 \mathrm{e}-50$ & 77 & $4.14742073168 \mathrm{e}-48$ & 78 & $1.78904091502 \mathrm{e}-46$ & 79 & $7.40817919355 \mathrm{e}-45$ \\
\hline 80 & $2.943700133510 \mathrm{e}-43$ & 81 & $1.122014888380 \mathrm{e}-41$ & 82 & $4.10061125513 \mathrm{e}-40$ & 83 & $1.43633249117 \mathrm{e}-38$ \\
\hline 84 & $4.81968183211 \mathrm{e}-37$ & 85 & $1.548559986510 \mathrm{e}-35$ & 86 & $4.76169394977 \mathrm{e}-34$ & 87 & $1.400502643990 \mathrm{e}-32$ \\
\hline 88 & $3.93774707023 \mathrm{e}-31$ & 89 & $1.05777224840 \mathrm{e}-29$ & 90 & $2.71295150626 \mathrm{e}-28$ & 91 & $6.63907833056 \mathrm{e}-27$ \\
\hline 92 & $1.549117947290 \mathrm{e}-25$ & 93 & $3.44390394051 \mathrm{e}-24$ & 94 & $7.28902479104 \mathrm{e}-23$ & 95 & $1.467519582110 \mathrm{e}-21$ \\
\hline 96 & $2.808147993620 \mathrm{e}-20$ & 97 & $5.10250621963 \mathrm{e}-19$ & 98 & $8.79548303360 \mathrm{e}-18$ & 99 & $1.43685110544 \mathrm{e}-16$ \\
\hline 100 & $2.22218436508 \mathrm{e}-15$ & 101 & $3.24998071837 \mathrm{e}-14$ & 102 & $4.489567612890 \mathrm{e}-13$ & 103 & $5.85079932579 \mathrm{e}-12$ \\
\hline 104 & $7.18370974878 \mathrm{e}-11$ & 105 & $8.29873790933 \mathrm{e}-10$ & 106 & $9.0070339192 \mathrm{e}-09$ & 107 & $9.1707380762 \mathrm{e}-08$ \\
\hline 108 & $8.7457189835 \mathrm{e}-07$ & 109 & 7.7990666196e-06 & 110 & $6.4924284455 \mathrm{e}-05$ & 111 & $5.036525894 \mathrm{e}-04$ \\
\hline 112 & $3.6345151422 \mathrm{e}-03$ & 113 & $2.4355146991 \mathrm{e}-02$ & 114 & $1.5129598801 \mathrm{e}-01$ & 115 & $8.699064420 \mathrm{e}-01$ \\
\hline 116 & $4.62298136 \mathrm{e}+00$ & 117 & $2.268127865 \mathrm{e}+01$ & 118 & $1.02629299 \mathrm{e}+02$ & 119 & $4.2781349 \mathrm{e}+02$ \\
\hline 120 & $1.6399167 \mathrm{e}+03$ & 121 & $5.7590488 \mathrm{e}+03$ & 122 & $1.839118 \mathrm{e}+04$ & 123 & $5.26980 \mathrm{e}+04$ \\
\hline 124 & $1.3266774 \mathrm{e}+05$ & 125 & $2.8583 \mathrm{e}+05$ & 126 & $5.2381 \mathrm{e}+05$ & 127 & $9.3264 \mathrm{e}+05$ \\
\hline 128 & $2.342 \mathrm{e}+06$ & 129 & $7.741 \mathrm{e}+06$ & 130 & $1.944 \mathrm{e}+07$ & 131 & $1.41 \mathrm{e}+07$ \\
\hline 132 & $-1.08 \mathrm{e}+08$ & 133 & $-4.566 \mathrm{e}+08$ & 134 & $-6.14 e+08$ & 135 & $1.1 \mathrm{e}+09$ \\
\hline 136 & $5.58 \mathrm{e}+09$ & 137 & $7.3 \mathrm{e}+09$ & 138 & $-6.6 e+09$ & 139 & $-3.37 e+10$ \\
\hline 140 & $-3.7 e+10$ & 141 & $5.5 e+09$ & 142 & $4.3 e+10$ & 143 & $2.7 \mathrm{e}+10$ \\
\hline 144 & $1.9 \mathrm{e}+09$ & & & & & & \\
\hline
\end{tabular}


TABle 9. Coefficients for $k=13$ truncating at $P=1699$, and using $\delta=10^{-12}$.

\begin{tabular}{|c|c|c|c|c|c|c|c|}
\hline$r$ & $c_{r}(13)$ & $r$ & $c_{r}(13)$ & $r$ & $c_{r}(13)$ & $r$ & $c_{r}(13)$ \\
\hline 0 & $2.58 \mathrm{e}-282$ & 1 & $4.33 \mathrm{e}-278$ & 2 & $3.60 \mathrm{e}-274$ & 3 & $1.97 \mathrm{e}-270$ \\
\hline 4 & $8.05 \mathrm{e}-267$ & 5 & $2.60 \mathrm{e}-263$ & 6 & $6.94 \mathrm{e}-260$ & 7 & $1.57 \mathrm{e}-256$ \\
\hline 8 & $3.08 \mathrm{e}-253$ & 9 & $5.31 \mathrm{e}-250$ & 10 & $8.16 \mathrm{e}-247$ & 11 & $1.13 \mathrm{e}-243$ \\
\hline 12 & $1.42 \mathrm{e}-240$ & 13 & $1.63 \mathrm{e}-237$ & 14 & $1.71 \mathrm{e}-234$ & 15 & $1.67 \mathrm{e}-231$ \\
\hline 16 & $1.51 \mathrm{e}-228$ & 17 & $1.27 \mathrm{e}-225$ & 18 & $9.96 \mathrm{e}-223$ & 19 & $7.34 \mathrm{e}-220$ \\
\hline 20 & $5.08 \mathrm{e}-217$ & 21 & $3.31 \mathrm{e}-214$ & 22 & $2.04 \mathrm{e}-211$ & 23 & $1.19 \mathrm{e}-208$ \\
\hline 24 & $6.56 \mathrm{e}-206$ & 25 & $3.43 \mathrm{e}-203$ & 26 & $1.71 \mathrm{e}-200$ & 27 & $8.11 \mathrm{e}-198$ \\
\hline 28 & $3.67 \mathrm{e}-195$ & 29 & $1.58 \mathrm{e}-192$ & 30 & $6.52 \mathrm{e}-190$ & 31 & $2.57 \mathrm{e}-187$ \\
\hline 32 & $9.69 \mathrm{e}-185$ & 33 & $3.50 \mathrm{e}-182$ & 34 & $1.21 \mathrm{e}-179$ & 35 & $4.04 \mathrm{e}-177$ \\
\hline 36 & $1.29 \mathrm{e}-174$ & 37 & $3.96 \mathrm{e}-172$ & 38 & $1.17 \mathrm{e}-169$ & 39 & $3.32 \mathrm{e}-167$ \\
\hline 40 & $9.068 \mathrm{e}-165$ & 41 & $2.387 \mathrm{e}-162$ & 42 & $6.054 \mathrm{e}-160$ & 43 & $1.480 \mathrm{e}-157$ \\
\hline 44 & $3.490 \mathrm{e}-155$ & 45 & $7.939 \mathrm{e}-153$ & 46 & $1.743 \mathrm{e}-150$ & 47 & $3.695 \mathrm{e}-148$ \\
\hline 48 & $7.563 \mathrm{e}-146$ & 49 & $1.496 \mathrm{e}-143$ & 50 & $2.858 \mathrm{e}-141$ & 51 & $5.280 \mathrm{e}-139$ \\
\hline 52 & $9.429 \mathrm{e}-137$ & 53 & $1.628 \mathrm{e}-134$ & 54 & $2.720 \mathrm{e}-132$ & 55 & $4.396 \mathrm{e}-130$ \\
\hline 56 & $6.874 \mathrm{e}-128$ & 57 & $1.040 \mathrm{e}-125$ & 58 & $1.524 \mathrm{e}-123$ & 59 & $2.160 \mathrm{e}-121$ \\
\hline 60 & $2.966 \mathrm{e}-119$ & 61 & $3.942 \mathrm{e}-117$ & 62 & $5.074 \mathrm{e}-115$ & 63 & $6.3253 \mathrm{e}-113$ \\
\hline 64 & $7.6365 \mathrm{e}-111$ & 65 & $8.9299 \mathrm{e}-109$ & 66 & $1.0115 \mathrm{e}-106$ & 67 & $1.1098 \mathrm{e}-104$ \\
\hline 68 & $1.1795 \mathrm{e}-102$ & 69 & $1.2144 \mathrm{e}-100$ & 70 & $1.2112 \mathrm{e}-98$ & 71 & $1.1702 \mathrm{e}-96$ \\
\hline 72 & $1.0952 \mathrm{e}-94$ & 73 & $9.9295 \mathrm{e}-93$ & 74 & $8.7200 \mathrm{e}-91$ & 75 & $7.4173 \mathrm{e}-89$ \\
\hline 76 & $6.11074 \mathrm{e}-87$ & 77 & $4.87569 \mathrm{e}-85$ & 78 & $3.76736 \mathrm{e}-83$ & 79 & $2.81878 \mathrm{e}-81$ \\
\hline 80 & $2.04204 \mathrm{e}-79$ & 81 & $1.43219 \mathrm{e}-77$ & 82 & $9.72340 \mathrm{e}-76$ & 83 & $6.38944 \mathrm{e}-74$ \\
\hline 84 & $4.063248 \mathrm{e}-72$ & 85 & $2.500254 \mathrm{e}-70$ & 86 & $1.488419 \mathrm{e}-68$ & 87 & $8.5708030 \mathrm{e}-67$ \\
\hline 88 & $4.7730131 \mathrm{e}-65$ & 89 & $2.5701193 \mathrm{e}-63$ & 90 & $1.3378668 \mathrm{e}-61$ & 91 & $6.7309343 \mathrm{e}-60$ \\
\hline 92 & $3.2721950 \mathrm{e}-58$ & 93 & $1.5367284 \mathrm{e}-56$ & 94 & $6.9700536 \mathrm{e}-55$ & 95 & $3.0523694 \mathrm{e}-53$ \\
\hline 96 & $1.29025276 \mathrm{e}-51$ & 97 & $5.26280865 \mathrm{e}-50$ & 98 & $2.0707478 \mathrm{e}-48$ & 99 & $7.8570340 \mathrm{e}-47$ \\
\hline 100 & $2.8738212 \mathrm{e}-45$ & 101 & $1.01291060 \mathrm{e}-43$ & 102 & $3.43894917 \mathrm{e}-42$ & 103 & $1.124213471 \mathrm{e}-40$ \\
\hline 104 & $3.53717984 \mathrm{e}-39$ & 105 & $1.07067892 \mathrm{e}-37$ & 106 & $3.11641068 \mathrm{e}-36$ & 107 & 8.71833921e-35 \\
\hline 108 & $2.34302639 \mathrm{e}-33$ & 109 & $6.04582505 \mathrm{e}-32$ & 110 & $1.49702508 \mathrm{e}-30$ & 111 & $3.55505913 \mathrm{e}-29$ \\
\hline 112 & $8.09182488 \mathrm{e}-28$ & 113 & $1.76422359 \mathrm{e}-26$ & 114 & $3.68197859 \mathrm{e}-25$ & 115 & $7.35070729 \mathrm{e}-24$ \\
\hline 116 & $1.40275907 \mathrm{e}-22$ & 117 & $2.55690377 \mathrm{e}-21$ & 118 & $4.44814493 \mathrm{e}-20$ & 119 & $7.37932244 \mathrm{e}-19$ \\
\hline 120 & $1.16640624 \mathrm{e}-17$ & 121 & $1.755027226 \mathrm{e}-16$ & 122 & $2.51133489 \mathrm{e}-15$ & 123 & $3.41411712 \mathrm{e}-14$ \\
\hline 124 & $4.40504845 \mathrm{e}-13$ & 125 & $5.38821792 \mathrm{e}-12$ & 126 & $6.24112402 \mathrm{e}-11$ & 127 & $6.83724634 \mathrm{e}-10$ \\
\hline 128 & $7.07544905 \mathrm{e}-09$ & 129 & $6.90733979 \mathrm{e}-08$ & 130 & $6.352697060 \mathrm{e}-07$ & 131 & $5.49643677 \mathrm{e}-06$ \\
\hline 132 & $4.4673281 \mathrm{e}-05$ & 133 & $3.40576382 \mathrm{e}-04$ & 134 & $2.4318473 \mathrm{e}-03$ & 135 & $1.6239805 \mathrm{e}-02$ \\
\hline 136 & $1.0128549 \mathrm{e}-01$ & 137 & $5.8923590 \mathrm{e}-01$ & 138 & $3.194017 \mathrm{e}+00$ & 139 & $1.611797 \mathrm{e}+01$ \\
\hline 140 & $7.566471 \mathrm{e}+01$ & 141 & $3.301742 \mathrm{e}+02$ & 142 & $1.337419 \mathrm{e}+03$ & 143 & $5.014434 \mathrm{e}+03$ \\
\hline 144 & $1.73025 \mathrm{e}+04$ & 145 & $5.43813 \mathrm{e}+04$ & 146 & $1.53198 \mathrm{e}+05$ & 147 & $3.7902 \mathrm{e}+05$ \\
\hline 148 & $8.147 \mathrm{e}+05$ & 149 & $1.6140 \mathrm{e}+06$ & 150 & $3.743 \mathrm{e}+06$ & 151 & $1.18 \mathrm{e}+07$ \\
\hline 152 & $3.577 \mathrm{e}+07$ & 153 & $6.04 \mathrm{e}+07$ & 154 & $-8.98 \mathrm{e}+07$ & 155 & $-8.78 \mathrm{e}+08$ \\
\hline 156 & $-2.1 \mathrm{e}+09$ & 157 & $1.30 \mathrm{e}+09$ & 158 & $2.1 \mathrm{e}+10$ & 159 & $5.2 \mathrm{e}+10$ \\
\hline 160 & $-9 e+09$ & 161 & $-3.6 e+11$ & 162 & $-8.7 e+11$ & 163 & $-5.2 \mathrm{e}+11$ \\
\hline 164 & $1.6 \mathrm{e}+12$ & 165 & $3.83 \mathrm{e}+12$ & 166 & $2.6 \mathrm{e}+12$ & 167 & $-9 \mathrm{e}+11$ \\
\hline 168 & $-1.65 \mathrm{e}+12$ & 169 & $-3.7 e+11$ & & & & \\
\hline
\end{tabular}


TABLE 10. For comparison, high precision values of $c_{r}(k)$, $4 \leq k \leq 13, r \leq 7$, computed using the program for method 1 of [CFKRS. The values here agree with those in Tables 29 computed using our cubic accelerant method with experimentally determined remainders, except they are occasionally slightly off in the last decimal place.

\begin{tabular}{|c|c|c|c|c|c|}
\hline$k$ & $r$ & $c_{r}(k)$ & $k$ & $r$ & $c_{r}(k)$ \\
\hline 4 & 0 & $.24650183919342273540799 \mathrm{e}-12$ & 9 & 0 & $.79201552383685290316 \mathrm{e}-113$ \\
\hline 4 & 1 & $.545014057311718655936 \mathrm{e}-10$ & 9 & 1 & $.36087438729455558616 \mathrm{e}-109$ \\
\hline 4 & 2 & $.5287729634791203113849 \mathrm{e}-8$ & 9 & 2 & $.80512962716760934894 \mathrm{e}-106$ \\
\hline 4 & 3 & $.29641143179993979459691 \mathrm{e}-6$ & 9 & 3 & $.11723624058166636900 \mathrm{e}-102$ \\
\hline 4 & 4 & $.10645950068128470513211 \mathrm{e}-4$ & 9 & 4 & $.12530058768923713471 \mathrm{e}-99$ \\
\hline 4 & 5 & $.2570298334242634023549 \mathrm{e}-3$ & 9 & 5 & $.10481427375523351016 \mathrm{e}-96$ \\
\hline 4 & 6 & $.426392161631169472187 \mathrm{e}-2$ & 9 & 6 & $.71456310032205157639 \mathrm{e}-94$ \\
\hline 4 & 7 & $.4894142451421601027126 \mathrm{e}-1$ & 9 & 7 & $.40821744596370636463 \mathrm{e}-91$ \\
\hline 5 & 0 & $.14160010206227312010 \mathrm{e}-23$ & 10 & 0 & $.35488849247730348098 \mathrm{e}-147$ \\
\hline 5 & 1 & $.73804127564944513060 \mathrm{e}-21$ & 10 & 1 & $.23576913310137009644 \mathrm{e}-143$ \\
\hline 5 & 2 & $.17797796235196529053 \mathrm{e}-18$ & 10 & 2 & $.77023366302575780180 \mathrm{e}-140$ \\
\hline 5 & 3 & $.26358866096607247583 \mathrm{e}-16$ & 10 & 3 & $.16494863440733411303 \mathrm{e}-136$ \\
\hline 5 & 4 & $.26840545349997485760 \mathrm{e}-14$ & 10 & 4 & $.26045194469316625626 \mathrm{e}-133$ \\
\hline 5 & 5 & $.19936413092498971803 \mathrm{e}-12$ & 10 & 5 & $.32336667784065596864 \mathrm{e}-130$ \\
\hline 5 & 6 & $.11184855124933629438 \mathrm{e}-10$ & 10 & 6 & $.32876514157441589044 \mathrm{e}-127$ \\
\hline 5 & 7 & $.48427975530448041655 \mathrm{e}-9$ & 10 & 7 & $.28147294699934449064 \mathrm{e}-124$ \\
\hline 6 & 0 & $.51294734091491911243 \mathrm{e}-39$ & 11 & 0 & $.124513138816594309 \mathrm{e}-186$ \\
\hline 6 & 1 & $.53067328099264442456 \mathrm{e}-36$ & 11 & 1 & $.116057289076806867 \mathrm{e}-182$ \\
\hline 6 & 2 & $.26079207711483512396 \mathrm{e}-33$ & 11 & 2 & $.533593692953085661 \mathrm{e}-179$ \\
\hline 6 & 3 & $.81016132157790177281 \mathrm{e}-31$ & 11 & 3 & $.161328064239033845 \mathrm{e}-175$ \\
\hline 6 & 4 & $.17861297380093099773 \mathrm{e}-28$ & 11 & 4 & $.360796891855797563 \mathrm{e}-172$ \\
\hline 6 & 5 & $.29743167108636063482 \mathrm{e}-26$ & 11 & 5 & $.636556626245602757 \mathrm{e}-169$ \\
\hline 6 & 6 & $.38877082911558678876 \mathrm{e}-24$ & 11 & 6 & $.922782528634884471 \mathrm{e}-166$ \\
\hline 6 & 7 & $.40922426140686293551 \mathrm{e}-22$ & 11 & 7 & $.113037084302453487 \mathrm{e}-162$ \\
\hline 7 & 0 & $.65822847876005499378 \mathrm{e}-59$ & 12 & 0 & $.26143756530064042 \mathrm{e}-231$ \\
\hline 7 & 1 & $.12041430555451865785 \mathrm{e}-55$ & 12 & 1 & $.33131354510381580 \mathrm{e}-227$ \\
\hline 7 & 2 & $.10621355717492716606 \mathrm{e}-52$ & 12 & 2 & $.20758311485643071 \mathrm{e}-223$ \\
\hline 7 & 3 & $.60172653760159300486 \mathrm{e}-50$ & 12 & 3 & $.85728395653185504 \mathrm{e}-220$ \\
\hline 7 & 4 & $.24606287673240130820 \mathrm{e}-47$ & 12 & 4 & $.26251165314413802 \mathrm{e}-216$ \\
\hline 7 & 5 & $.77390121665211526042 \mathrm{e}-45$ & 12 & 5 & $.63569771378458374 \mathrm{e}-213$ \\
\hline 7 & 6 & $.19478649494952357456 \mathrm{e}-42$ & 12 & 6 & $.12679941162107841 \mathrm{e}-209$ \\
\hline 7 & 7 & $.40307684926363697065 \mathrm{e}-40$ & 12 & 7 & $.21425932836667245 \mathrm{e}-206$ \\
\hline 8 & 0 & $.18704421601168844202 \mathrm{e}-83$ & 13 & 0 & $.2577425553942569 \mathrm{e}-281$ \\
\hline 8 & 1 & $.55702193651787573285 \mathrm{e}-80$ & 13 & 1 & $.4326313738224894 \mathrm{e}-277$ \\
\hline 8 & 2 & $.80727983790767114280 \mathrm{e}-77$ & 13 & 2 & $.3596648214485737 \mathrm{e}-273$ \\
\hline 8 & 3 & $.75876025208717340817 \mathrm{e}-74$ & 13 & 3 & $.1974403388369282 \mathrm{e}-269$ \\
\hline 8 & 4 & $.52002464291967325362 \mathrm{e}-71$ & 13 & 4 & $.8051097934153342 \mathrm{e}-266$ \\
\hline 8 & 5 & $.27705098412043360903 \mathrm{e}-68$ & 13 & 5 & $.2601086374395006 \mathrm{e}-262$ \\
\hline 8 & 6 & $.11944832708048773794 \mathrm{e}-65$ & 13 & 6 & $.6934795975034907 \mathrm{e}-259$ \\
\hline 8 & 7 & $.42839952698747409380 \mathrm{e}-63$ & 13 & 7 & $.1569263805950391 \mathrm{e}-255$ \\
\hline
\end{tabular}


TABLE 11. The value of $r$ for each $k$ for which the corresponding term in (52) contributes the dominant amount when $T=10^{8}$.

\begin{tabular}{|c||c|c|c|c|c|c|c|c|c|c|c|c|c|}
\hline$k$ & 1 & 2 & 3 & 4 & 5 & 6 & 7 & 8 & 9 & 10 & 11 & 12 & 13 \\
\hline$r$ & 0 & 0 & 2 & 6 & 11 & 18 & 26 & 37 & 49 & 64 & 80 & 99 & 119 \\
\hline
\end{tabular}

TABLE 12. The values of $\operatorname{Data}_{k}(T)$ and $\operatorname{Conjecture}_{k}(T)$ at $T=$ 100000000.64, the first zero after $10^{8}$. We also display, in the last two columns, the values of the averages over all 1000 values of $T_{j}$ : $\frac{1}{1000} \sum_{j=1}^{1000} \operatorname{Data}_{k}\left(T_{j}\right)$, and $\frac{1}{1000} \sum_{j=1}^{1000}$ Conjecture $_{k}\left(T_{j}\right)$.

\begin{tabular}{|c|c|c||c|c|}
\hline$k$ & Data $_{k}(T)$ & Conjecture $_{k}(T)$ & $(58)$ & $(59)$ \\
\hline 1 & 1673723690.436 & 1673723498.495 & 62463107.03367 & 62463106.44834 \\
2 & 637388343407. & 637389923500. & 22091815715.8 & 22091815007.3 \\
3 & $8.04585314342 \mathrm{e}+14$ & $8.04581403344 \mathrm{e}+14$ & $2.54969941363 \mathrm{e}+13$ & $2.54969410053 \mathrm{e}+13$ \\
4 & $1.7376480696 \mathrm{e}+18$ & $1.7374512576 \mathrm{e}+18$ & $5.0233293703 \mathrm{e}+16$ & $5.0234406051 \mathrm{e}+16$ \\
5 & $5.0837678819 \mathrm{e}+21$ & $5.0816645028 \mathrm{e}+21$ & $1.3436072658 \mathrm{e}+20$ & $1.3439894636 \mathrm{e}+20$ \\
6 & $1.815301994 \mathrm{e}+25$ & $1.813639687 \mathrm{e}+25$ & $4.400401104 \mathrm{e}+23$ & $4.406152513 \mathrm{e}+23$ \\
7 & $7.480512969 \mathrm{e}+28$ & $7.468884126 \mathrm{e}+28$ & $1.66796929 \mathrm{e}+27$ & $1.674460675 \mathrm{e}+27$ \\
8 & $3.43851173 \mathrm{e}+32$ & $3.43090327 \mathrm{e}+32$ & $7.0665341 \mathrm{e}+30$ & $7.13020297 \mathrm{e}+30$ \\
9 & $1.72388578 \mathrm{e}+36$ & $1.71918466 \mathrm{e}+36$ & $3.26838252 \mathrm{e}+34$ & $3.32617445 \mathrm{e}+34$ \\
10 & $9.2785049 \mathrm{e}+39$ & $9.251733 \mathrm{e}+39$ & $1.6228859 \mathrm{e}+38$ & $1.6729912 \mathrm{e}+38$ \\
11 & $5.2991086 \mathrm{e}+43$ & $5.2863072 \mathrm{e}+43$ & $8.5447722 \mathrm{e}+41$ & $8.967058 \mathrm{e}+41$ \\
12 & $3.182548 \mathrm{e}+47$ & $3.179455 \mathrm{e}+47$ & $4.726347 \mathrm{e}+45$ & $5.076008 \mathrm{e}+45$ \\
13 & $1.995625 \mathrm{e}+51$ & $1.999377 \mathrm{e}+51$ & $2.726982 \mathrm{e}+49$ & $3.013406 \mathrm{e}+49$ \\
\hline
\end{tabular}

TABLE 13. The standard deviations of 900 values of the remainder terms, and smoothed remainder terms, for $1 \leq k \leq 13$. Specifically, we computed the standard deviation for the values of (53), $101 \leq$ $j \leq 1000$, and of (57), for $101 \leq J \leq 1000$.

\begin{tabular}{|c|c|c|}
\hline$k$ & Standard deviation & Standard deviation, smoothed \\
\hline 1 & $5.0337 \mathrm{e}-07$ & $4.4433 \mathrm{e}-08$ \\
2 & $7.6993 \mathrm{e}-06$ & $2.7833 \mathrm{e}-07$ \\
3 & $5.9228 \mathrm{e}-05$ & $6.5080 \mathrm{e}-06$ \\
4 & 0.00032311 & $8.9497 \mathrm{e}-05$ \\
5 & 0.0012292 & 0.00046265 \\
6 & 0.0034752 & 0.0015118 \\
7 & 0.0079677 & 0.0038227 \\
8 & 0.015741 & 0.0081774 \\
9 & 0.027867 & 0.015496 \\
10 & 0.045375 & 0.026757 \\
11 & 0.069216 & 0.042914 \\
12 & 0.10024 & 0.064832 \\
13 & 0.1392 & 0.093254 \\
\hline
\end{tabular}



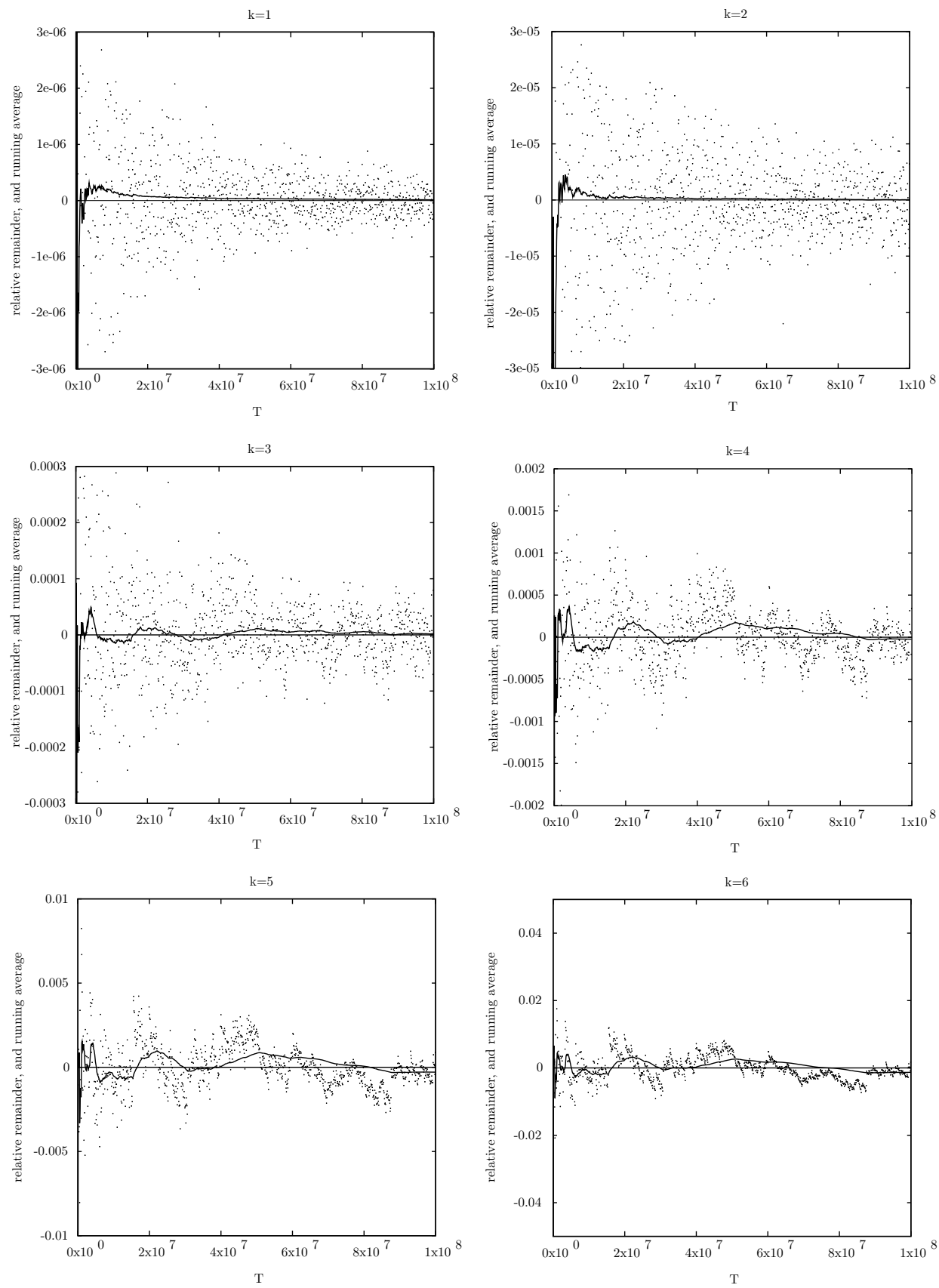

Figure 1. Plot of the relative remainder (53), depicted as dots, and running average (solid curve) of the remainder (57), for 1000 values of $T$, and $1 \leq k \leq 6$. Horizontal axis is $T$. 

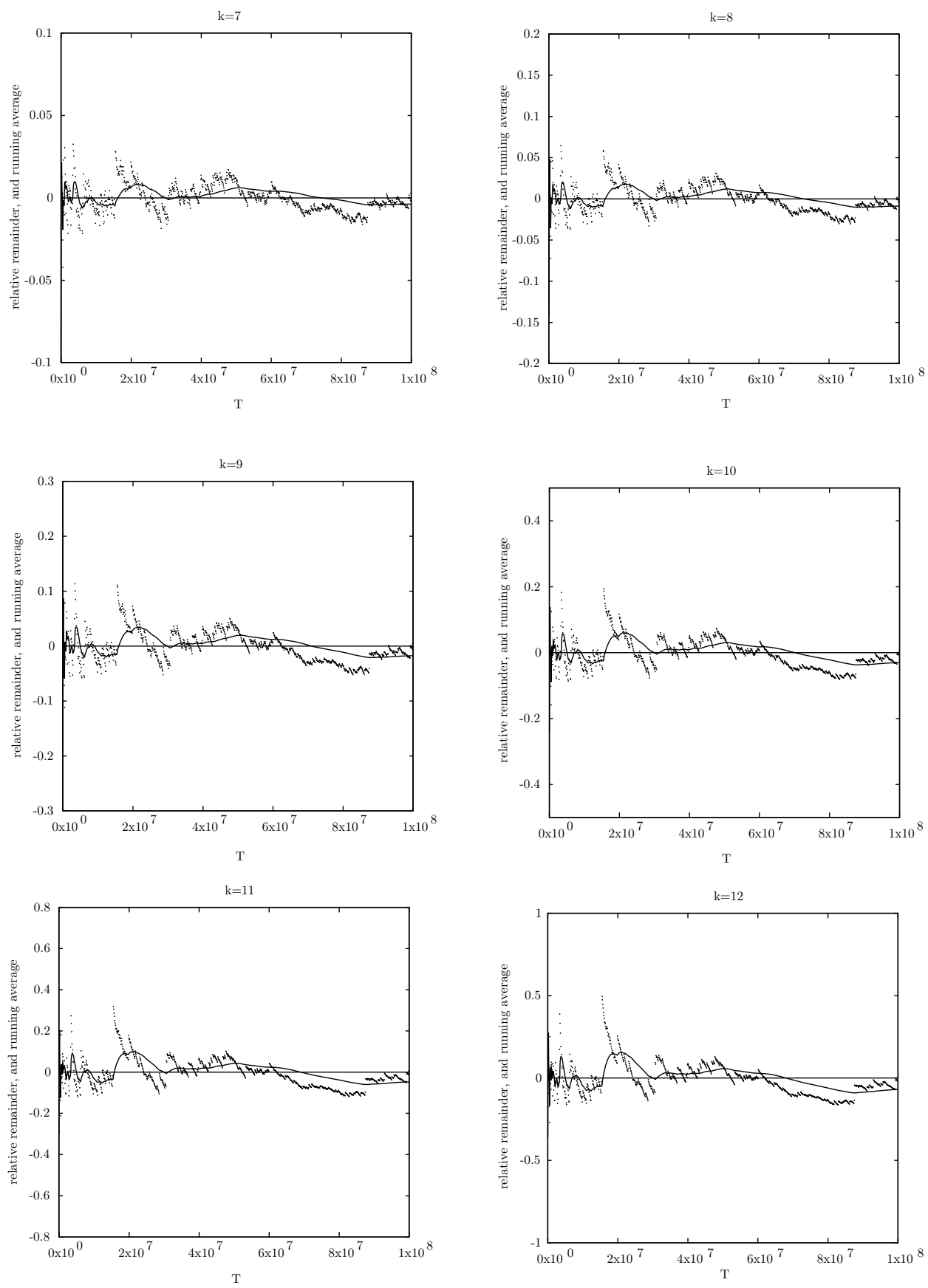

Figure 2. Plot of the relative remainder (53), and running average (solid curve) of the remainder (57), for 1000 values of $T$, and $7 \leq k \leq 12$. Horizontal axis is $T$. 


\section{ACKNOWLEDGEMENT}

The authors would like to thank the referee for his insightful feedback.

\section{REFERENCES}

[Ba] David H. Bailey, Tanh-Sinh High-Precision Quadrature, Jan 2006; LBNL-60519.

[BLJ] David H. Bailey, Karthik Jeyabalan, and Xiaoye S. Li, A comparison of three high-precision quadrature schemes, Experiment. Math. 14 (2005), no. 3, 317-329. MR2172710 (2006e:65047)

[Br] Richard P. Brent, Algorithms for Minimization without Derivatives, Prentice-Hall Inc., Englewood Cliffs, NJ., 1973. Prentice-Hall Series in Automatic Computation. MR0339493 (49 \#4251)

[CFKRS] J. B. Conrey, D. W. Farmer, J. P. Keating, M. O. Rubinstein, and N. C. Snaith, Integral moments of L-functions, Proc. London Math. Soc. (3) 91 (2005), no. 1, 33-104, DOI 10.1112/S0024611504015175. MR2149530(2006j:11120)

[CFKRS2] J. B. Conrey, D. W. Farmer, J. P. Keating, M. O. Rubinstein, and N. C. Snaith, Lower order terms in the full moment conjecture for the Riemann zeta function, J. Number Theory 128 (2008), no. 6, 1516-1554, DOI 10.1016/j.jnt.2007.05.013. MR2419176 (2009b:11139)

[DGH] Adrian Diaconu, Dorian Goldfeld, and Jeffrey Hoffstein, Multiple Dirichlet series and moments of zeta and L-functions, Compositio Math. 139 (2003), no. 3, 297-360, DOI 10.1023/B:COMP.0000018137.38458.68. MR2041614(2005a:11124)

[E] H. Edwards, Riemann's Zeta Function, Academic Press, 1974.

[FHLPZ] Laurent Fousse, Guillaume Hanrot, Vincent Lefèvre, Patrick Pélissier, and Paul Zimmermann, MPFR: a multiple-precision binary floating-point library with correct rounding, ACM Trans. Math. Software 33 (2007), no. 2, Art. 13, 15, DOI 10.1145/1236463.1236468. MR2326955(2008e:65157)

[H-B] D. R. Heath-Brown, The fourth power moment of the Riemann zeta function, Proc. London Math. Soc. (3) 38 (1979), no. 3, 385-422, DOI 10.1112/plms/s3-38.3.385. MR.532980 (81f:10052)

[HO] Ghaith A. Hiary and Andrew M. Odlyzko, The zeta function on the critical line: numerical evidence for moments and random matrix theory models, Math. Comp. 81 (2012), no. 279, 1723-1752, DOI 10.1090/S0025-5718-2011-02573-1. MR2904600

[HR] Ghaith A. Hiary and Michael O. Rubinstein, Uniform asymptotics for the full moment conjecture of the Riemann zeta function, J. Number Theory 132 (2012), no. 4, 820868, DOI 10.1016/j.jnt.2011.09.009. MR2887620

[I] A. E. Ingham, Mean-value theorems in the theory of the Riemann zeta-function, Proc. London Math. Soc. S2-27, no. 1, 273, DOI 10.1112/plms/s2-27.1.273. MR.1575391

[R] M. O. Rubinstein, lcalc - the L-function calculator, http://code.google.com/p/ $1-\mathrm{calc} /$.

University of Waterloo, Department of Pure Mathematics, 200 University Avenue W, Waterloo, ON N2L 3G1 CANAdA.

E-mail address: michael.o.rubinstein@gmail.com

University of Waterloo, Department of Pure Mathematics, 200 University Avenue W, WATERLOO, ON N2L 3G1 CANADA.

E-mail address: syamagishi@uwaterloo.ca 